\title{
ON THE INTEGRAL COHOMOLOGY RING OF TORIC ORBIFOLDS AND SINGULAR TORIC VARIETIES
}

\author{
ANTHONY BAHRI, SOUMEN SARKAR, AND JONGBAEK SONG
}

\begin{abstract}
We examine the integral cohomology rings of certain families of $2 n$-dimensional orbifolds $X$ that are equipped with a well-behaved action of the $n$-dimensional real torus. These orbifolds arise from two distinct but closely related combinatorial sources, namely from characteristic pairs $(Q, \lambda)$, where $Q$ is a simple convex $n$-polytope and $\lambda$ a labelling of its facets, and from $n$ dimensional fans $\Sigma$. In the literature, they are referred as toric orbifolds and singular toric varieties respectively. Our first main result provides combinatorial conditions on $(Q, \lambda)$ or on $\Sigma$ which ensure that the integral cohomology groups $H^{*}(X)$ of the associated orbifolds are concentrated in even degrees. Our second main result assumes these condition to be true, and expresses the graded ring $H^{*}(X)$ as a quotient of an algebra of polynomials that satisfy an integrality condition arising from the underlying combinatorial data. Also, we compute several examples.
\end{abstract}

\section{Contents}

1. Introduction

2. A retraction of simple polytopes

3. Toric orbifolds and orbifold lens spaces

3.1. Toric orbifolds

3.2. Invariant subspaces

3.3. Orbifold lens spaces

4. Vanishing odd degree homology and torsion freeness

5. Cohomology ring of toric orbifolds 16

5.1. Weighted Stanley-Reisner ring 17

5.2. Piecewise algebra and cohomology ring 20

6. Example: Orbifold Hirzebruch varieties 22

References

\section{INTRODUCTION}

There are several advantages to studying topological spaces whose integral cohomology groups $H^{*}(X)$ are torsion-free and concentrated in even degrees; for example, their complex $K$-theory and complex cobordism groups may be deduced

2010 Mathematics Subject Classification. Primary 14M25, 55N91, 57R18; Secondary 13F55, $52 \mathrm{~B} 11$.

Key words and phrases. toric orbifold, quasitoric orbifold, toric variety, Lens space, equivariant cohomology, Stanley-Reisner ring, piecewise polynomial. 
immediately, because the appropriate Atiyah-Hirzebruch spectral sequences collapse for dimensional reasons. For convenience, we call such spaces even, where integral coefficients are understood unless otherwise stated. Our fundamental aim is to identify certain families of even spaces within the realms of toric topology, and to explain how their evenness leads to a description of the Borel equivariant cohomology rings $H_{T}^{*}(X)$, and thence to the multiplicative structure of $H^{*}(X)$.

Many even spaces arise from complex geometry, and have been of major importance since the early 20th century. They range from complex projective spaces and Grassmannian manifolds, to Thom spaces of complex vector bundles over other even spaces. Examples of the latter include stunted projective spaces, which play an influential and enduring role in homotopy theory, and certain restricted families of weighted projective spaces. In fact every weighted projective space is even, thanks to a beautiful and somewhat surprising result of Kawasaki Kaw73, whose calculations lie behind one of our main works in Section 4. In the literature, weighted projective spaces have been viewed as singular toric varieties or as toric orbifolds which we shall define in Section 3, and our results may be interpreted as an investigation of their generalizations within either context.

We begin in Section 2 by introducing a sequence $\left\{B_{k}\right\}$ of polytopal complexes whose initial term is a simple polytope $Q$ and the final term is a vertex of $Q$. We define the sequence inductively by the rule stated as $\$$ in Section 2 which is motivated by several spaces called invariant subspaces, and orbifold lens spaces sitting inside the given toric orbifold.

In Section 3 , we summarize the theory of toric orbifolds $X=X(Q, \lambda)$, as constructed from an $n$-dimensional simple convex polytope $Q$ and an $\mathcal{R}$-characteristic function $\lambda$ from its facets to $\mathbb{Z}^{n}$. The combinatorial data $(Q, \lambda)$ is called an $\mathcal{R}$ characteristic pair associated to the given toric orbifold. The notion of invariant subspaces and orbifold lens spaces follow from $(Q, \lambda)$, which we shall explain in the following subsections. Moreover, for each polytopal complex $B$ which appears in a retraction sequence, the $\mathcal{R}$-characteristic function $\lambda$ may be used to associate a finite group $G_{B}(v)$, see (4.8), to certain vertices $v$ called free vertices in $B$, and to define the collection

$$
\left\{\left|G_{B}(v)\right| \mid v \text { is a free vertex in } B\right\} .
$$

Interest in toric orbifolds was stimulated by Davis and Januszkiewicz DJ91, who saw them as natural extensions to their own smooth toric manifolds?. They proved that toric manifolds are always even; however, the best comparable statement for toric orbifolds is due to Poddar and the second author [PS10] who showed that, in general, they are only even over the rationals. We introduce our main result of the first part of this paper in Section 4 as follows.

Theorem 1.1. Given any toric orbifold $X(Q, \lambda)$, assume that the gcd of the collection (1.1) is 1 for each $B$ which appears in a retraction sequence with $\operatorname{dim} B>1$; then $X$ is even.

The proof employs a cofiber sequence involving orbifold lens spaces, which are generalization of lens complexes, introduced by Kawasaki Kaw73. Furthermore, Theorem 1.1 automatically applies to weighted projective spaces.

\footnotetext{
${ }^{1}$ In the literature, these orbifolds are sometimes called quasitoric orbifolds

${ }^{2}$ They are renamed in $\mathrm{BP} 02$ as quasitoric manifolds.
} 
In Section [5, we restrict our emphasis to projective toric orbifolds, which are realized as toric varieties whose details are admirably presented by Cox, Little and Schenck in their encyclopedic book [CLS11]. Every such variety $X_{\Sigma}$ is encoded by a fan $\Sigma$ in $\mathbb{R}^{n}$, and admits a canonical action by the $n$-dimensional real torus $T^{n}$. If $\Sigma$ is smooth, then the underlying geometry guarantees that $X_{\Sigma}$ is always even. Moreover, it is true that the Borel equivariant cohomology ring $H_{T}^{*}\left(X_{\Sigma}\right)$ is isomorphic to the Stanley-Reisner ring $\mathcal{S R}[\Sigma]$, which is also concentrated in even degrees, and $H^{*}\left(X_{\Sigma}\right)$ is its quotient by a linear ideal determined by (5.2). It is important to note that $\mathcal{S R}[\Sigma]$ is isomorphic to the ring $\mathcal{P} \mathcal{P}[\Sigma]$ of integral piecewise polynomials on $\Sigma$, for any smooth fan.

For a particular class of singular examples, a comparable description of the ring $H^{*}\left(X_{\Sigma}\right)$ was given in BFR09, as follows. If $\Sigma$ is polytopal and $X_{\Sigma}$ is even, then $H^{*}\left(X_{\Sigma}\right)$ is the quotient of $\mathcal{P} \mathcal{P}[\Sigma]$ by the ideal generated by all global polynomials. It is no longer possible to use the Stanley-Reisner ring, which only agrees with $\mathcal{P} \mathcal{P}[\Sigma]$ over the rationals. In these circumstances, when $X_{\Sigma}$ is a toric variety over a polytopal fan, we have a major incentive to develop criteria which test whether or not it is even. There also remains the significant problem of presenting $\mathcal{P} \mathcal{P}[\Sigma]$ by generators and relations, as exemplified by the calculation for the weighted projective space $\mathbb{C P}_{(1,2,3,4)}^{3}$ in [BFR09, §4]. So the aim of Section [5 is to find an alternative description for the ring of piecewise polynomials. It is accomplished by defining the weighted Stanley-Reisner ring $w \mathcal{S R}[\Sigma]$, which turned out to be a subring of $\mathcal{S R}[\Sigma]$, consisting of polynomials that satisfy an integrality condition, see Definition 5.2 The main result of the Section 5 combines Theorem 1.1 and Theorem [5.4, as follows.

Theorem 1.2. Given any polytopal fan $\Sigma$ in $\mathbb{R}^{n}$, assume that the corresponding $\mathcal{R}$-characteristic pair $(Q, \lambda)$ satisfies the hypothesis of Theorem 1.1; then $X_{\Sigma}$ is even, and there exists an isomorphism

$$
H^{*}\left(X_{\Sigma}\right) \cong w \mathcal{S R}[\Sigma] / \mathcal{J}
$$

of graded rings, where $\mathcal{J}$ is an ideal of linear relations determined by the generators of rays of $\Sigma$.

So our combinatorial condition on the fan allows us to give an explicit description of the integral cohomology ring of $X_{\Sigma}$.

Several natural questions present themselves for future discussion. For example, Sections 3 and 5 may be linked more closely by establishing a common framework for toric orbifolds and toric varieties over non-smooth polytopal fans. The theory of multifans is an obvious candidate, but we have been unable to identify an associated ring of piecewise polynomials with sufficient clarity. However, the third author with Darby and Kuroki DKS has recently proposed a definition of piecewise polynomials on an orbifold torus graph, which does allow those two objects to be dealt with simultaneously.

In view of our opening remarks, another reasonable challenge is to extend our study to the complex $K$-theory and complex cobordism of toric orbifolds. This program was suggested by work of Harada, Henriques and Holm [HHH05, and begun in HRHW15] by the adoption of a categorical approach to piecewise structures; but overall progress has been limited to a small subfamily of weighted projective spaces, and much further work is required. However, some progress have done by the second author and Uma $\underline{\mathrm{SU}}$. 
Acknowledgments. We extend our sincere gratitude to Nigel Ray. Most properly, his name belongs among those of the authors. The significance of his mathematical contribution to the results is most certainly not reflected accurately by the omission of his name. Among his many other contributions to the paper is the concise and elegant introduction. We thank Mikiya Masuda and Haozhi Zeng for pointing out a gap in the proof of Theorem 2.12 of the previous version, and $\mathrm{Li}$ Cai for helpful comments about retraction sequences. We are also grateful for the hospitality of University of Calgary in July, 2015.

This work has been supported in part by Simons Foundation Grants 210368 and 426160. The first author acknowledges the award of research leave from Rider University and is grateful also for the hospitality of the Princeton University Mathematics Department during the spring of 2015. The second author would like to thank Pacific Institute for the Mathematical Sciences and University of Regina for financial support. Lastly, the third author was supported by Basic Science Research Program through the National Research Foundation of Korea (NRF) funded by the Korea government (MSIP) (No. NRF-2016R1A2B4010823). He also would like to thank his advisor Prof. Dong Youp Suh for his encouragements and support throughout the project.

\section{A RETRACTION OF SIMPLE POLYTOPES}

In this section, we introduce a natural way of retracting a simple polytope $Q$ to a point, which we call a retraction sequence. For each polytope, there are finitely many such retractions, enabling us to develop a sufficient condition for torsion freeness in the homology of toric orbifolds in the following section. The operation itself is motivated by several spaces which arise in a toric orbifold by decomposing the orbit space. We shall explain this topological interpretation in Section 3. This section is devoted to give the combinatorial definition and properties of retraction sequences. We begin by introducing the definition of a polytopal complex.

Definition 2.1. [Zie95, Definition 5.1] A polytopal complex $\mathcal{C}$ is a finite collection of polytopes in $\mathbb{R}^{n}$ satisfying:

(1) if $E$ is a face of $F$ and $F \in \mathcal{C}$ then $E \in \mathcal{C}$,

(2) if $E, F \in \mathcal{C}$ then $E \cap F$ is a face of both $E$ and $F$.

Let $|\mathcal{C}|=\bigcup_{F \in \mathcal{C}} F$ be the underlying set of $\mathcal{C}$.

The elements of $\mathcal{C}$ are called faces and the zero dimensional faces of $\mathcal{C}$ are called vertices. We denote the set of vertices of $\mathcal{C}$ by $V(|\mathcal{C}|)$. The dimension of $\mathcal{C}$ or $|\mathcal{C}|$ is the maximum of the dimension of its faces. Given a simple polytope $Q$, let $\mathcal{C}(Q)$ be the collection of all faces of $Q$ and $\mathscr{F}(Q)$ the collection of all facets of $Q$. Then, $\mathcal{C}(Q)$ is a polytopal complex and $|\mathcal{C}(Q)|$ is homeomorphic to $Q$ as manifolds with corners. Through out this paper, we always assume that $\ell:=|V(Q)|$, the number of vertices of $Q, m:=|\mathscr{F}(Q)|$, the number of facets of $Q$ and $n:=\operatorname{dim} Q$.

Now, given an $n$-dimensional simple polytope $Q$, we construct a sequence of triples $\left\{\left(B_{k}, E_{k}, b_{k}\right)\right\}_{k=1}^{\ell}$, which we call a retraction sequence of $Q$. First, we define $B_{1}=Q=E_{1}$ and $b_{1} \in V\left(B_{1}\right)$. The second term $\left(B_{2}, E_{2}, b_{2}\right)$ is defined as follows. Consider a subcollection

$$
\mathcal{C}_{2}=\left\{E \in \mathcal{C}(Q) \mid b_{1} \notin V(E)\right\}
$$




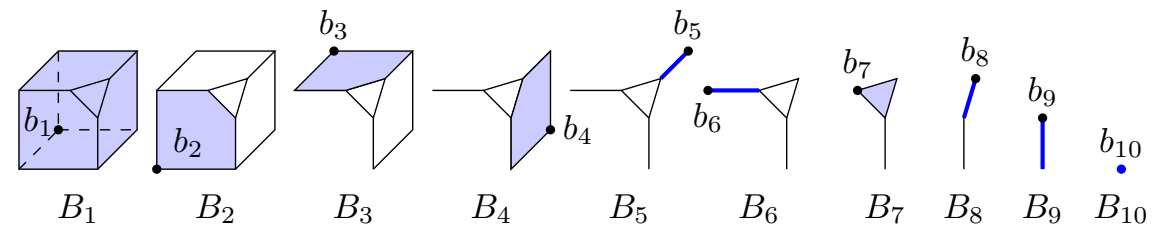

FiguRE 1. A retraction sequence of a vertex cut of the cube.

of $\mathcal{C}(Q)$. Then, $\mathcal{C}_{2}$ is an $(n-1)$-dimensional polytopal complex. We define $B_{2}$ by the underlying set $\left|\mathcal{C}_{2}\right|$ of $\mathcal{C}_{2}$. We choose vertex $b_{2}$ of $B_{2}$ such that $b_{2}$ has a neighborhood diffeomorphic to $\mathbb{R}_{>0}^{N}$ as manifold with corners, for some $1 \leq N \leq \operatorname{dim} B_{2}$ and let $E_{2}$ be the unique $N$-dimensional face of $B_{2}$ containing $b_{2}$. Notice that, in this case, $N=n-1$ and we have $n$ many different choices of $b_{2}$ because $Q$ is an $n$-dimensional simple polytope.

Next, we construct the sequence inductively. Given $\left(B_{k}, E_{k}, b_{k}\right)$, the next term $\left(B_{k+1}, E_{k+1}, b_{k+1}\right)$ is defined as follows. First we consider a polytopal complex

$$
\mathcal{C}_{k+1}=\left\{E \in \mathcal{C}_{k} \mid b_{k} \notin V(E)\right\} .
$$

Then, $B_{k+1}$ is defined by its underlying set $\left|\mathcal{C}_{k+1}\right|$. We choose a vertex $b_{k+1}$ in $V\left(B_{k+1}\right)$ satisfying the following condition:

( ) " $b_{k+1}$ has a neighborhood homeomorphic to $\mathbb{R}_{\geq 0}^{N}$ as manifold with corners, for some $N \in\left\{1, \ldots, \operatorname{dim} B_{k+1}\right\} "$ and $E_{k+1}$ defined to be a unique face of $B_{k+1}$ containing $b_{k+1}$ with $\operatorname{dim} E_{k+1}=N$.

Definition 2.2. We call a vertex $v$ in $B_{k}$ a free vertex, if it has a neighborhood in $B_{k}$ that is diffeomorphic to $\mathbb{R}_{>0}^{N}$ as manifold with corners, for some $N \in$ $\left\{1, \ldots, \operatorname{dim} B_{k}\right\}$. We denote the set of free vertices in $B_{k}$ by $F V\left(B_{k}\right)$.

The proof of Proposition 2.3 below guarantees the existence of free vertices at each step. Finally, the sequence stops if the sequence reaches a vertex, i.e., $B_{\ell}=$ $E_{\ell}=b_{\ell} \in V(Q)$. Essentially, we can think of a retraction sequence as an iterated choice of free vertices at each step. Figure 1 shows an example of retraction sequence for the vertex cut of a cube, where the colored face of each $B_{k}$ indicates $E_{k}$ for $k=1, \ldots, 10$.

Proposition 2.3. Every simple polytope has at least one retraction sequence.

Proof. We begin by following the argument of [DJ91, Proposition 3.1]. First, we realize $Q$ as a convex polytope in $\mathbb{R}^{n}$ and choose a vector $u \in \mathbb{R}^{n}$ such that

$$
\langle u, v\rangle \neq\langle u, w\rangle \text {, whenever } v \neq w \in V(Q) \subset \mathbb{R}^{n},
$$

with respect to the Euclidean inner product $\langle$,$\rangle . Let e:=e(v w)$ be the oriented edge with the initial vertex $i(e)=v$ and the terminal vertex $t(e)=w$. Here, the direction of $e(v w)$ is given by the following rule:

$$
i(e)=v \text { and } t(e)=w \text {, if and only if }\langle u, v\rangle<\langle u, w\rangle,
$$

which makes the one skeleton of $Q$ into a directed graph.

Let $\operatorname{ind}(v)$ be the number of inward edges at $v$ and we call $\operatorname{ind}(v)$ the index of $v$ (with respect to the choice of generic vector $u$ ). Then, for each face $E \subset Q$, there 
exists the unique vertex $v$ of $E$ having the maximal index among the vertices in $E$. Moreover, $E$ is locally diffeomorphic to $\mathbb{R}_{\geq 0}^{i n d(v)}$ around $v$. Conversely, given a vertex $v \in V(Q)$, there exists a unique face $E_{v}^{-}$such that $\operatorname{dim} E_{v}=\operatorname{ind}(v)$.

Let $\left\{b_{k}\right\}_{k=1}^{\ell}$ be a sequence of vertices in $Q$ determined by

$$
\left\langle u, b_{1}\right\rangle>\left\langle u, b_{2}\right\rangle>\cdots>\left\langle u, b_{\ell}\right\rangle \text {. }
$$

Notice that $i n d\left(b_{1}\right)=n=\operatorname{dim} Q$, and $i n d\left(b_{\ell}\right)=0$. Now, we claim that the following sequence

$$
\left\{\left(B_{k}:=\bigcup_{j \geq k} E_{b_{j}}, E_{b_{k}}, b_{k}\right)\right\}_{k=1, \ldots, \ell},
$$

where $E_{b_{k}}$ is a unique face containing $b_{k}$ with $\operatorname{dim} E_{b_{k}}=\operatorname{ind}\left(b_{k}\right)$, is a retraction sequence of $Q$. Indeed, for each $k \in\{1, \ldots, \ell-1\}$, we have $\left\langle u, b_{k}\right\rangle>\langle u, v\rangle$, for all $v \in V\left(B_{k}\right) \backslash\left\{b_{k}\right\}$. Hence, there are no outgoing edges from $b_{k}$ in $B_{k}$, which implies that $b_{k}$ has a neighborhood in $E_{b_{k}} \subseteq B_{k}$ homeomorphic to $\mathbb{R}_{\geq 0}^{\text {ind }\left(b_{k}\right)}$ as manifold with corners.

We denote by $\mathfrak{R}(Q)$ the set of all retraction sequences of $Q$ and by $\mathfrak{B}(Q)$ the set of all possible $B_{i}$ 's which appear in $\mathfrak{R}(Q)$. Evidently, both $\mathfrak{R}(Q)$ and $\mathfrak{B}(Q)$ are finite sets, because we have finitely many choice of free vertices at each step.

Remark 2.4. The retraction sequence has a strong relation with shelling of a simplicial complex. We are preparing an independent article [BSS] about the exact correspondence and some other interesting properties.

\section{TORIC ORBIFOLDS AND ORBIFOLD LENS SPACES}

In this section we recall the characteristic pairs $(Q, \lambda)$ of [DJ91] and PS10, and explain the way in which they are used to construct toric orbifolds $X=X(Q, \lambda)$. If $\lambda$ obeys Davis and Januszkiewicz's condition $(*)$ (see [DJ91, page 423]), then $X$ is smooth and even; so one of the main goals of this paper is to establish Theorem 1.1. which focuses on singular cases, and states a sufficient condition for the orbifold $X$ to be even. In this section, to complete the proof of Theorem 1.1, we commandeer two additional types of spaces, namely the invariant subspaces of $X$ which arise as the preimage of faces via the orbit map, and the orbifold lens spaces that arise as quotients of odd dimensional spheres by the actions of certain finite groups associated to $\lambda$.

3.1. Toric orbifolds. In this subsection, we discuss a combinatorial definition of toric orbifolds. Let $Q$ be an $n$-dimensional simple convex polytope in $\mathbb{R}^{n}$ and $\mathscr{F}(Q)=\left\{F_{1}, \ldots, F_{m}\right\}$ the codimension one faces of $Q$ which are called facets.

Definition 3.1. A function $\lambda: \mathscr{F}(Q) \rightarrow \mathbb{Z}^{n}$ is called a rational characteristic function (or $\mathcal{R}$-characteristic function) for $Q$ if it satisfies the following condition:

$$
\left\{\lambda\left(F_{i_{1}}\right), \ldots, \lambda\left(F_{i_{k}}\right)\right\} \text { is linearly independent, whenever } \bigcap_{j=1}^{k} F_{i_{j}} \neq \varnothing .
$$

We denote $\lambda_{i}=\lambda\left(F_{i}\right)$ and call it an $\mathcal{R}$-characteristic vector assigned to the facet $F_{i}$. The pair $(Q, \lambda)$ is called an $\mathcal{R}$-characteristic pair. 
Remark 3.2. (1) In the literature about toric manifolds, the pair $(Q, \lambda)$ satisfying the condition $(*)$ in DJ91, p. 423] is called a characteristic pair.

(2) For convenience, we usually express an $\mathcal{R}$-characteristic function $\lambda$ as an $(n \times m)$-matrix $\Lambda$ by listing $\lambda_{i}$ 's as column vectors. We call $\Lambda$ an $\mathcal{R}$ characteristic matrix associated to $\lambda$.

(3) It is easy to check that it suffices to satisfy the linearly independence at each vertex which is an intersection of $n$ facets.

One canonical example of such function can be given by a simple lattice polytope which is a convex hull of finitely many points in the integer lattice $\mathbb{Z}^{n} \subset \mathbb{R}^{n}$ and simple. Namely, we can naturally assign an $\mathcal{R}$-characteristic vector the primitive normal vector on each facet of a simple lattice polytope. In Section 5 we shall see this again as primitive vectors of 1-dimensional cones in a normal fan associated to a simple lattice polytope.

For $x \in Q$, we denote by $E(x)$ the face of $Q$ which contains $x$ in its interior. If $E(x)$ is a face of codimension $k$, then it is a unique intersection of $k$ facets $F_{i_{1}}, \ldots, F_{i_{k}}$. We also denote by $T_{E(x)}$ the subtorus of standard $n$-dimensional torus $T^{n}$, determined by $\lambda_{i_{1}}, \ldots, \lambda_{i_{k}}$. To be more precise, we may regard the target space $\mathbb{Z}^{n}$ of $\lambda$ as the $\mathbb{Z}$-submodule of the Lie algebra of $T^{n}$, and $T_{E(x)}$ is the torus generated by the exponential image of the lines determined by the $\mathcal{R}$-characteristic vectors $\lambda_{i_{1}}, \ldots, \lambda_{i_{k}}$.

Now, we define an equivalence relation $\sim$ on the product $T^{n} \times Q$ by

$$
(t, x) \sim_{\lambda}(s, y) \text { if and only if } x=y \text { and } t^{-1} s \in T_{E(x)} .
$$

The quotient space

$$
X(Q, \lambda)=\left(T^{n} \times Q\right) / \sim_{\lambda}
$$

has an orbifold structure with a natural $T^{n}$-action induced by the group operation, see Section 2 in PS10. Clearly, the orbit space of $T^{n}$-action on $X(Q, \lambda)$ is $Q$. Let

$$
\pi: X(Q, \lambda) \rightarrow Q, \quad \pi\left([t, x]_{\sim_{\lambda}}\right)=x
$$

be the orbit map, where $[t, x]_{\sim_{\lambda}}$ is the equivalence class of $(t, x)$ with respect to $\sim_{\lambda}$. The space $X(Q, \lambda)$ is called the toric orbifold associated to the combinatorial pair $(Q, \lambda)$.

In analyzing the orbifold structure of $X(Q, \lambda)$, Poddar and Sarkar, PS10, Subsection 2.2], gave an axiomatic definition of toric orbifolds, which generalizes the axiomatic definition of toric manifolds of [DJ91.

3.2. Invariant subspaces. In this subsection, we study the $\mathcal{R}$-characteristic pair of some invariant subspaces of $X(Q, \lambda)$. Let $E=F_{i_{1}} \cap \cdots \cap F_{i_{k}}$ be a face of $Q$, where $F_{i_{1}}, \ldots, F_{i_{k}}$ are facets. We can define a natural projection

$$
\rho_{E}: \mathbb{Z}^{n} \rightarrow \mathbb{Z}^{n} /\left(\left(\operatorname{span}\left\{\lambda_{i_{1}}, \ldots, \lambda_{i_{k}}\right\} \otimes_{\mathbb{Z}} \mathbb{R}\right) \cap \mathbb{Z}^{n}\right),
$$

where the target space is isomorphic to $\mathbb{Z}^{n-k}$, because $\left(\operatorname{span}\left\{\lambda_{i_{1}}, \ldots, \lambda_{i_{k}}\right\} \otimes_{\mathbb{Z}} \mathbb{R}\right) \cap \mathbb{Z}^{n}$ is a rank $k$ direct summand of $\mathbb{Z}^{n}$. Notice that the rank of the target space of $\rho_{E}$ is same as the dimension of $E$. We consider $E$ as an independent simple polytope, and denote the set of facets of $E$ by

$$
\mathscr{F}(E)=\left\{E \cap F_{j} \mid F_{j} \in \mathscr{F}(Q) \text { and } j \neq i_{1}, \ldots, i_{k} \text {, and } E \cap F_{j} \neq \varnothing\right\} .
$$

Now, the map $\rho_{E}$ together with $\lambda$ yields an $\mathcal{R}$-characteristic function

$$
\lambda_{E}: \mathscr{F}(E) \rightarrow \mathbb{Z}^{n-k} \text {, }
$$




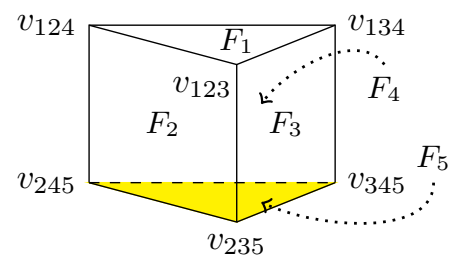

$$
\Lambda=\left[\begin{array}{ccccc}
\lambda_{1} & \lambda_{2} & \lambda_{3} & \lambda_{4} & \lambda_{5} \\
3 & 2 & -1 & -1 & 0 \\
3 & -1 & -1 & 2 & 0 \\
5 & 0 & -2 & 2 & 1
\end{array}\right]
$$

Figure 2.

on $E$ defined for $\lambda_{E}\left(E \cap F_{j}\right)$ to be the primitive vector of $\left(\rho_{E} \circ \lambda\right)\left(F_{j}\right)$. Indeed, the condition (3.1) naturally follows from $\lambda$.

Hence, we get an $\mathcal{R}$-characteristic pair $\left(E, \lambda_{E}\right)$ from $(Q, \lambda)$, which yields another toric orbifold

$$
X\left(E, \lambda_{E}\right):=\left(T^{n-k} \times E\right) / \sim_{\lambda_{E}},
$$

where the equivalence relation $\sim_{\lambda_{E}}$ defined in a manner similar to (3.2).

Proposition 3.3. [PS10, Section 2.3] Let $\pi: X(Q, \lambda) \rightarrow Q$ and $\left(E, \lambda_{E}\right)$ be as above. Then, $\pi^{-1}(E)$ is a $T^{n}$-invariant suborbifold. Moreover, it is a toric orbifold homeomorphic to $X\left(E, \lambda_{E}\right)$ as a topological space.

The second assertion of the above proposition follows from the fact that the circle subgroups determined by $\lambda_{E}\left(E \cap F_{j}\right)$ and $\left(\rho_{E} \circ \lambda\right)\left(F_{j}\right)$, respectively, are identical. We also remark that the torus $T^{n-k}$ acting on $X\left(E, \lambda_{E}\right)$ can be identified with the image of the map

$$
\bar{\rho}_{E}: T^{n} \rightarrow T^{n-k},
$$

which is induced from the map $\rho_{E}$.

Example 3.4. Suppose we have an $\mathcal{R}$-characteristic pair $(Q, \lambda)$ as described in Figure 2, Notice that $Q$ is a 3 -dimensional polytope with 5 facets, say $\mathscr{F}(Q)=$ $\left\{F_{1}, \ldots, F_{5}\right\}$. Here, we assume that the target space $\mathbb{Z}^{3}$ of $\lambda$ is generated by the standard basis $\left\{e_{1}, e_{2}, e_{3}\right\}$. We choose $E$ to be the facet $F_{5}$. So, $k=1$ and $n-k=2$. Then, the projection

$$
\rho_{E}: \mathbb{Z}^{3} \rightarrow \mathbb{Z}^{3} /\left\langle e_{3}\right\rangle=\left\langle e_{1}, e_{2}, e_{3}\right\rangle /\left\langle e_{3}\right\rangle \cong \mathbb{Z}^{2}
$$

is onto the first two coordinates. The facets of $E$ are $F_{2} \cap E, F_{3} \cap E$ and $F_{4} \cap E$. Hence, the map

$$
\lambda_{E}:\left\{F_{2} \cap E, F_{3} \cap E, F_{4} \cap E\right\} \longrightarrow \mathbb{Z}^{2}
$$

is defined by

$$
\begin{aligned}
& \lambda_{E}\left(F_{2} \cap E\right)=\rho_{E}\left(\lambda\left(F_{2}\right)\right)=(2,-1)=2 e_{1}-e_{2}, \\
& \lambda_{E}\left(F_{3} \cap E\right)=\rho_{E}\left(\lambda\left(F_{3}\right)\right)=(-1,-1)=-e_{1}-e_{2}, \\
& \lambda_{E}\left(F_{4} \cap E\right)=\rho_{E}\left(\lambda\left(F_{4}\right)\right)=(-1,2)=-e_{1}+2 e_{2} .
\end{aligned}
$$

The orbifold corresponding to $\left(E, \lambda_{E}\right)$ is known to be a fake weighted projective space with weight $(1,1,1)$. We refer [Buc08] and Kas09] for the details of fake weighted projective space. 
3.3. Orbifold lens spaces. Here, we introduce a generalization of lens complexes and study their homology groups. Let $\Delta^{n-1}$ be the $(n-1)$-dimensional simplex and $\mathscr{F}\left(\Delta^{n-1}\right)=\left\{F_{1}, \ldots, F_{n}\right\}$ the facets of $\Delta^{n-1}$. We begin by introducing the following definition.

Definition 3.5. A function $\xi: \mathscr{F}\left(\Delta^{n-1}\right) \rightarrow \mathbb{Z}^{n}$ is called an $\mathcal{L}$-characteristic function on $\Delta^{n-1}$ if $\left\{\xi\left(F_{1}\right), \ldots, \xi\left(F_{n}\right)\right\}$ is linearly independent. We set $\xi_{i}:=\xi\left(F_{i}\right)$ for $i=1, \ldots, n$.

Now, we define an equivalence relation $\sim_{\xi}$ on $T^{n} \times \Delta^{n-1}$ as follows:

$$
(t, x) \sim_{\xi}(s, y) \text { if and only if } x=y \text { and } t^{-1} s \in T_{F(x)},
$$

where $F(x)$ is the face containing $x$ in its interior and $T_{F(x)}$ denotes the subtorus of $T^{n}$ determined by $\xi_{i_{1}}, \ldots, \xi_{i_{k}}$, if $F(x)=F_{i_{1}} \cap \cdots \cap F_{i_{k}}$. The pair $\left(\Delta^{n-1}, \xi\right)$ together with the equivalence relation $\sim_{\xi}$ yields the following quotient space:

$$
L\left(\Delta^{n-1}, \xi\right):=T^{n} \times \Delta^{n-1} / \sim_{\xi},
$$

which we call the orbifold lens space associated to $\left(\Delta^{n-1}, \xi\right)$.

Proposition 3.6. The orbifold lens space $L\left(\Delta^{n-1}, \xi\right)$ is homeomorphic to the quotient space of the $(2 n-1)$-dimensional sphere $S^{2 n-1}$ by the action of a finite group $G_{\xi}:=\mathbb{Z}^{n} / \operatorname{span}\left\{\xi_{1}, \ldots, \xi_{n}\right\}$.

Proof. The proof is essentially same as the proof of [SS13, Proposition 2.3].

Remark 3.7. (1) In SS13, the function $\xi$ is called a hyper-characteristic function if the submodule generated by $\left\{\xi\left(F_{i_{1}}\right), \ldots, \xi\left(F_{i_{k}}\right)\right\}$ is a direct summand of $\mathbb{Z}^{n+1}$ of rank $k$, whenever $F_{i_{1}} \cap \cdots \cap F_{i_{k}}$ is nonempty. In particular, if $\left\{\xi\left(F_{i_{1}}\right), \ldots, \xi\left(F_{i_{n}}\right)\right\}$ is a linearly independent set, then it becomes an $\mathcal{L}$ characteristic function.

(2) The action of $G_{\xi}$ is induced from the standard $T^{n}$ - action on $S^{2 n-1} \subset \mathbb{C}^{n}$.

(3) The order $\left|G_{\xi}\right|$ of $G_{\xi}$ is exactly same as the determinant of the $n \times n$ matrix $\left[\begin{array}{l|l|l}\xi_{1} & \cdots & \xi_{n}\end{array}\right]$.

Proposition 3.6 leads us the following lemma.

Lemma 3.8. Let $p_{1}, \ldots, p_{r}$ be the prime factors of $\left|G_{\xi}\right|$. Then,

$$
H_{j}\left(L\left(\Delta^{n-1}, \xi\right)\right)=\left\{\begin{array}{ll}
\mathbb{Z} & \text { if } j=0,2 n-1 \\
G_{j} & \text { if } 1 \leq j \leq 2 n-2
\end{array},\right.
$$

where $G_{j}=\left(\mathbb{Z} / p_{1}^{a_{j_{1}}} \mathbb{Z}\right) \oplus \cdots \oplus\left(\mathbb{Z} / p_{r}^{a_{j_{r}}} \mathbb{Z}\right)$ for some non-negative integers $a_{1}, \ldots, a_{r}$.

Proof. We see $H_{0}\left(L\left(\Delta^{n-1}, \xi\right)\right) \cong \mathbb{Z}$ trivially. The isomorphism $H_{2 n-1}\left(L\left(\Delta^{n}, \xi\right)\right) \cong$ $\mathbb{Z}$ follows because $G_{\xi}$ action on $S^{2 n-1}$ is induced from the standard action of $T^{n}$ on $S^{2 n-1} \subset \mathbb{C}^{n}$, which is orientation preserving. For $j \in\{1, \ldots, 2 n-2\}$, recall the following isomorphism which can be obtained from the classical result for an action of a finite group $G$ on a locally compact Hausdorff space $X$ :

$$
H^{*}(X / G ; \mathbf{k}) \cong H^{*}(X ; \mathbf{k})^{G},
$$

where $\mathbf{k}$ is a field of characteristic zero or prime to $|G|$, see [Bor60, III.2].

We apply the isomorphism (3.8) to the orbifold lens space $L\left(\Delta^{n-1}, \xi\right) \cong S^{2 n-1} / G_{\xi}$. Since $H^{j}\left(S^{2 n-1} ; \mathbf{k}\right)^{G_{\xi}}=0$ for $j=1, \ldots 2 n-2$, the claim is proved by the universal coefficient theorem. 

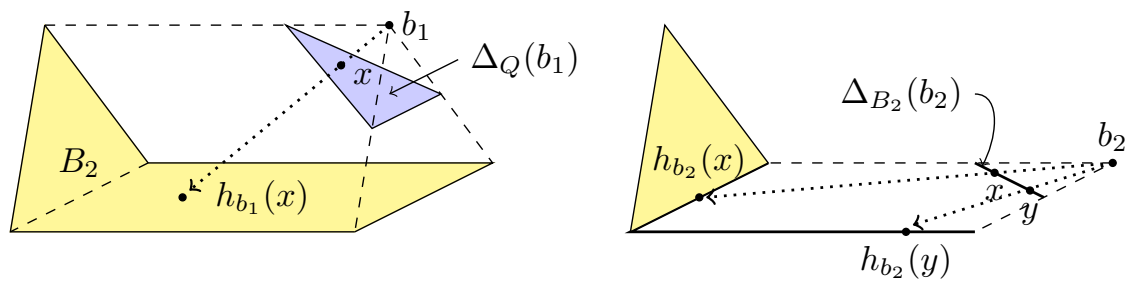

FiguRE 3. The geometric interpretation of a retraction sequence.

Toric orbifolds, invariant subspaces, and orbifold lens spaces motivate the definition of retraction sequences which we introduced in the previous section. For a vertex $v \in V(Q)$, let $B_{2}$ be the union of all faces in $Q$ which does not contain $v$. Next, we consider a hyperplane

$$
H(v):=\left\{x \in \mathbb{R}^{n} \mid\left\langle x, p_{v}\right\rangle=q_{v}\right\},
$$

where $\langle$,$\rangle denotes the Euclidean inner product, p_{v} \in \mathbb{R}^{n}$ and $q_{v} \in \mathbb{R}$ are chosen in such a way that

- $\left\{x \in \mathbb{R}^{n} \mid\left\langle x, p_{v}\right\rangle+q_{v} \geq 0\right\} \cap V(Q)=\{v\}$,

- $\left\{x \in \mathbb{R}^{n} \mid\left\langle x, p_{v}\right\rangle+q_{v} \leq 0\right\} \cap V(Q)=V(Q) \backslash\{v\}$.

Then, $\Delta_{Q}(v):=Q \cap H(v)$ is an $(n-1)$-dimensional simplex, because $Q$ is a simple polytope of dimension $n$, see Figure 3 .

An $\mathcal{L}$-characteristic pair arises naturally from an $\mathcal{R}$-characteristic pair $(Q, \lambda)$ for each vertex $v$ of $Q$. Indeed, if $v=F_{j_{1}} \cap \cdots \cap F_{j_{n}}$, we denote the set of facets of $\Delta_{Q}(v)$ by

$$
\mathscr{F}\left(\Delta_{Q}(v)\right)=\left\{\Delta_{Q}(v) \cap F_{j_{1}}, \ldots, \Delta_{Q}(v) \cap F_{j_{n}}\right\} .
$$

Now we define a function

$$
\xi_{Q, v}: \mathscr{F}\left(\Delta_{Q}(v)\right) \rightarrow \mathbb{Z}^{n},
$$

by $\xi_{Q, v}\left(\Delta_{Q}(v) \cap F_{j_{r}}\right)=\lambda\left(F_{j_{r}}\right), r=1, \ldots, n$. Notice that $\operatorname{dim} \Delta_{Q}(v)=n-1$, but the rank of target space is $n$. Since $\left\{\lambda\left(F_{i_{1}}\right) \ldots, \lambda\left(F_{i_{n}}\right)\right\}$ is a linearly independent set, the function $\xi_{Q, v}$ is an $\mathcal{L}$-characteristic function on $\Delta_{Q}(v)$.

\section{VANISHING ODD DEGREE HOMOLOGY AND TORSION FREENESS}

Now, we combine the ingredients which we introduced in the previous sections to derive a sufficient condition for vanishing odd degree cohomology of toric orbifolds. In particular, let $X(Q, \lambda)$ be a toric orbifold and the triple $\left\{\left(B_{k}, E_{k}, b_{k}\right)\right\}_{k=1}^{\ell}$ be a retraction sequence of $Q$. Given an $n$-dimensional polytope $Q$, we begin by defining the following map

$$
h_{b_{1}}: \Delta_{Q}\left(b_{1}\right) \rightarrow B_{2}=\bigcup\left\{E \mid E \text { is face of } Q, b_{1} \notin V(E)\right\}
$$

by $h_{b_{1}}(x)=B_{2} \cap$ (line passing through $x$ and $\left.b_{1}\right)$, where $\Delta_{Q}\left(b_{1}\right)$ is an $(n-1)$ dimensional simplex. The map $h_{b_{1}}$ is well-defined, because $Q$ is convex. The left picture of Figure 3 shows the map $h_{b_{1}}$ when $Q$ is a prism.

Define a map

$$
f_{b_{1}}: T^{n} \times \Delta_{Q}\left(b_{1}\right) \rightarrow \bigcup_{E: \text { face of } B_{2}} T^{\operatorname{dim} E} \times E,
$$


by $f_{b_{1}}(t, x)=\left(\bar{\rho}_{E}(t), h_{b_{1}}(x)\right)$, where $\bar{\rho}_{E}$ is defined in (3.6). This induces the the map

$$
\bar{f}_{b_{1}}: L\left(\Delta_{Q}\left(b_{1}\right), \xi_{Q, b_{1}}\right) \rightarrow \bigcup_{E: \text { face of } B_{2}} X\left(E, \lambda_{E}\right),
$$

where $\xi_{Q, b_{1}}$ is an $\mathcal{L}$-characteristic function defined in (3.10). This map is welldefined from the proof of the following proposition.

Proposition 4.1. The following diagram commutes:

$$
\begin{gathered}
T^{n} \times \Delta_{Q}\left(b_{1}\right) \stackrel{f_{b_{1}}}{\longrightarrow} \bigcup_{E: \text { face of } B_{2}}\left(T^{\operatorname{dim} E} \times E\right) \\
\qquad \begin{array}{l}
\mid / \sim_{\xi_{Q}, b_{1}} \\
L\left(\Delta_{Q}\left(b_{1}\right), \xi_{Q, b_{1}}\right) \stackrel{\bar{f}_{b_{1}}}{\longrightarrow}
\end{array} \bigcup_{E: \text { face of } B_{2}} X\left(E, \lambda_{E}\right) \longrightarrow X(Q, \lambda),
\end{gathered}
$$

where the equivalence relations $\sim_{\xi_{Q, b_{1}}}$ and $\sim_{\lambda_{E}}$ are defined similarly as in (3.7) and (3.2), respectively. Moreover, the bottom row is a cofiber sequence. i.e., $X(Q, \lambda)$ is homotopy equivalent to the mapping cone $c\left(\bar{f}_{b_{1}}\right)$ of the map $\bar{f}_{b_{1}}$.

Proof. We first show that the map $\bar{f}_{b_{1}}$ is well-defined. Suppose we choose two different representatives, say $[t, x]_{\sim_{\xi_{Q}, b_{1}}}$ and $[s, y]_{\sim_{\xi_{Q}, b_{1}}}$ in $L\left(\Delta_{Q}\left(b_{1}\right), \xi_{Q, b_{1}}\right)$. Then $x=y$, so $h_{b_{1}}(x)=h_{b_{1}}(y)$. Moreover, if $x \in \Delta_{Q}\left(b_{1}\right) \cap F$ for some face $F$ of $Q$, then $h_{b_{1}}(x) \in F \cap E$ for some face $E$ of $B_{2}$. Hence the map $\bar{\rho}_{E}$ sends the subtorus $T_{F(x)}$ of $T^{n}$ to $T_{E\left(h_{b_{1}}(x)\right)}$ the subtorus of $T^{\operatorname{dim} E}$. Since the map $\bar{\rho}_{E}$ is a homomorphism, if $t^{-1} s \in T_{F(x)}$, then

$$
\bar{\rho}_{E}(t)^{-1} \bar{\rho}_{E}(s)=\bar{\rho}_{E}\left(t^{-1} s\right) \in T_{E\left(h_{b_{1}}(x)\right)} .
$$

Let $C \Delta_{Q}\left(b_{1}\right)$ be the cone on $\Delta_{Q}\left(b_{1}\right)$ in $Q$ with the cone point $b_{1}$. Then, we can decompose $Q$ into two part as follows:

$$
Q=C \Delta_{Q}\left(b_{1}\right) \cup_{\Delta_{Q}\left(b_{1}\right)} \overline{Q \backslash C \Delta_{Q}\left(b_{1}\right)} .
$$

Now, we define a continuous surjective map

$$
g_{b_{1}}: \overline{Q \backslash C \Delta_{Q}\left(b_{1}\right)} \rightarrow B_{2}
$$

in a manner similar to (4.1). We use it to define a straight line homotopy by

$$
\phi: \overline{Q \backslash C \Delta_{Q}\left(b_{1}\right)} \times I \rightarrow \overline{Q \backslash C \Delta_{Q}\left(b_{1}\right)},(x, u) \mapsto(1-u) x+u \cdot g_{b_{1}}(x),
$$

which preserves the face structure. Thus, $\phi$ induces a homotopy

$$
\hat{\phi}:\left(T^{n} \times \overline{Q \backslash C \Delta_{Q}\left(b_{1}\right)}\right) / \sim_{\lambda} \times I \rightarrow\left(T^{n} \times \overline{Q \backslash C \Delta_{Q}\left(b_{1}\right)}\right) / \sim_{\lambda}
$$

defined by

$$
\left([t, x]_{\sim_{\lambda}}, u\right) \mapsto[t, c(x, u)]_{\sim_{\lambda}} .
$$

Note that at $u=0$ the map $\hat{\phi}$ is identity and at $u=1$ the image of $\hat{\phi}$ is $\pi^{-1}\left(B_{2}\right)$.

Then,

$$
\begin{aligned}
X(Q, \lambda) & =\pi^{-1}\left(C \Delta_{Q}\left(b_{1}\right)\right) \cup_{L\left(\Delta_{Q}\left(b_{1}\right), \xi_{Q, b_{1}}\right)} \pi^{-1}\left(\overline{Q \backslash C \Delta_{Q}\left(b_{1}\right)}\right) \\
& \simeq C\left(L\left(\Delta_{Q}\left(b_{1}\right), \xi_{Q, b_{1}}\right)\right) \cup_{L\left(\Delta_{Q}\left(b_{1}\right), \xi_{Q, b_{1}}\right)} \pi^{-1}\left(B_{2}\right) \\
& \simeq c\left(\bar{f}_{b_{1}}\right) .
\end{aligned}
$$

Hence, the result follows. 
Now the following isomorphisms are straightforward from the cofiber sequence.

$$
\begin{aligned}
H_{*}\left(X(Q, \lambda), \pi^{-1}\left(B_{2}\right)\right) & \cong H_{*}\left(C\left(L\left(\Delta_{Q}\left(b_{1}\right), \xi_{Q, b_{1}}\right)\right), \pi^{-1}\left(B_{2}\right)\right) \\
& \cong \widetilde{H}_{*-1}\left(L\left(\Delta_{Q}\left(b_{1}\right), \xi_{Q, b_{1}}\right)\right) .
\end{aligned}
$$

Those two isomorphisms come from the excision and the long exact sequence of the pair, respectively.

So far, we have considered the $B_{1}(=Q)$ and $B_{2}$ which is the second term of a retraction sequence starting by choosing $b_{1} \in F V(Q)=V(Q)$. However, we can apply the similar arguments to each pair $B_{i}$ and $B_{i+1}$ in a retraction sequence. This leads us the following Lemma whose proof is essentially same as that of Proposition 4.1. Before we state the lemma, we first set up the notations: Given a retraction sequence $\left\{\left(B_{k}, E_{k}, b_{k}\right)\right\}_{k=1}^{\ell}$ of $Q$,

- $\Delta_{E_{k}}\left(b_{k}\right):=E_{k} \cap H\left(b_{k}\right)=B_{k} \cap H\left(b_{k}\right)$ : the simplex obtained by cutting the vertex $b_{k}$ from $B_{k}$.

- $\xi_{E_{k}, b_{k}}$ : an $\mathcal{L}$-characteristic function on $\Delta_{E_{k}}\left(b_{k}\right)$ defined in a similar manner to (3.10) induced from $\lambda_{E_{k}}$.

- The map

$$
\bar{f}_{b_{k}}: L\left(\Delta_{E_{k}}\left(b_{k}\right), \xi_{E_{k}, b_{k}}\right) \rightarrow \bigcup_{E: \text { face of } B_{k+1}} X\left(E, \lambda_{E}\right)=\pi^{-1}\left(B_{k+1}\right)
$$

is defined similarly to (4.3) by regarding $E_{k}$ as a simple polytope.

The right hand side of Figure 3 illustrates the case of the 3 -dimensional prism. The argument above extends to prove the following lemma.

Lemma 4.2. The sequence

$$
L\left(\Delta_{E_{k}}\left(b_{k}\right), \xi_{E_{k}, b_{k}}\right) \stackrel{\bar{f}_{b_{k}}}{\longrightarrow} \pi^{-1}\left(B_{k+1}\right) \longrightarrow \pi^{-1}\left(B_{k}\right)
$$

is a cofiber sequence. Moreover, $H_{*}\left(\pi^{-1}\left(B_{k}\right), \pi^{-1}\left(B_{k+1}\right)\right) \cong \widetilde{H}_{*-1}\left(L\left(\Delta_{E_{k}}\left(b_{k}\right), \xi_{b_{k}}\right)\right)$.

Recall from the Remark 3.7 that an $\mathcal{L}$-characteristic function $\xi: \mathscr{F}\left(\Delta^{n-1}\right) \rightarrow \mathbb{Z}^{n}$ defines a finite abelian group $\mathbb{Z}^{n} / \operatorname{im}(\xi)$. An $\mathcal{R}$-characteristic pair $(Q, \lambda)$ induces an $\mathcal{R}$-characteristic pair $\left(E, \lambda_{E}\right)$ as in (3.5) for any face $E$ of $Q$. Let $E$ be a $k$ dimensional face of $Q(k \leq n)$ and $v \in V(E)$. Then, $\Delta_{E}(v):=E \cap H(v)$ is a $(k-1)$-simplex. These give us an $\mathcal{L}$-characteristic function

$$
\xi_{E, v}: \mathscr{F}\left(\Delta_{E}(v)\right) \rightarrow \mathbb{Z}^{k}
$$

which is defined in a similar manner to (3.10) associated to $\lambda_{E}: \mathscr{F}(E) \rightarrow \mathbb{Z}^{k}$ and $v \in V(E)$. This $\mathcal{L}$-characteristic function defines the finite group

$$
G_{E}(v):=\mathbb{Z}^{k} / \operatorname{im}\left(\xi_{E, v}\right) .
$$

If $G_{E}(v)$ is trivial, we call a point $\pi^{-1}(v)$ in $\pi^{-1}(E) \cong X\left(E, \lambda_{E}\right)$ a smooth point, otherwise a singular point, where $\pi: X(Q, \lambda) \rightarrow Q$ is the orbit map defined in (3.3).

Furthermore, for each $B \in \mathfrak{B}(Q)$ and a free vertex $v \in F V(B)$, there exists a unique maximal face, say $E_{v}$, of $B$ containing $v$. Hence, for each $B \in \mathfrak{B}(Q)$, we denote by

$$
G_{B}(v):=G_{E_{v}}(v),
$$

whenever $v$ is a free vertex in $B$. 
Proposition 4.3. Given a vertex $v \in V(Q)$, let $E$ and $E^{\prime}$ be two faces containing $v$ such that $E$ is a face of $E^{\prime}$. Then, $\left|G_{E}(v)\right|$ divides $\left|G_{E^{\prime}}(v)\right|$.

Proof. From Proposition 3.3, we may assume that $E^{\prime}=Q$ without loss of generality. Suppose that $E$ is a face of $Q$ with codimension $k$. For convenience, we further assume that $E=F_{1} \cap \cdots \cap F_{k}$ and $v=F_{1} \cap \cdots \cap F_{k} \cap F_{k+1} \cap \cdots \cap F_{n}$, where $F_{i}$ 's are facets of $Q$.

From (3.10) and (4.7), we have $G_{Q}(v)=\mathbb{Z}^{n} /\left\langle\lambda\left(F_{1}\right), \ldots, \lambda\left(F_{n}\right)\right\rangle$ and $G_{E}(v)=$ $\mathbb{Z}^{k} /\left\langle\lambda_{E}\left(E \cap F_{k+1}\right), \ldots, \lambda_{E}\left(E \cap F_{n}\right)\right\rangle$. Now we consider the following composition

$$
\mathbb{Z}^{n} \stackrel{\rho_{E}}{\longrightarrow} \mathbb{Z}^{k} \longrightarrow \mathbb{Z}^{k} /\left\langle\lambda_{E}\left(E \cap F_{k+1}\right), \ldots, \lambda_{E}\left(E \cap F_{n}\right)\right\rangle
$$

where the map $\rho_{E}$ is defined in (3.4) and the second map is the natural surjection determined by (3.5). Observe that the kernel of the previous composition contains $\left\langle\lambda\left(F_{1}\right), \ldots, \lambda\left(F_{n}\right)\right\rangle$. Hence, we get a surjective group homomorphism from $G_{Q}(v)$ to $G_{E}(v)$. The result follows from the Lagrange's theorem in group theory.

We are now in a position to prove Theorem 1.1

Proof of Theorem 1.1. We prove the claim by the induction on the number of vertices of $B \in \mathfrak{B}(Q)$. First, notice that when the retraction sequence reaches an edge or a union of edges, say $B_{s}$, then $\pi^{-1}\left(B_{s}\right)$ is $\mathbb{C P}^{1}$ or homotopic to a finite wedge of $\mathbb{C P}^{1}$, which implies that $H_{*}\left(\pi^{-1}\left(B_{s}\right)\right)$ is torsion free and concentrated in even degrees. Therefore, if $|V(B)| \leq 2$ for $B \in \mathfrak{B}(Q)$, then the claim is true.

Now we assume that $\pi^{-1}(B)$ is even for $B \in \mathfrak{B}(Q)$ with $|V(B)| \leq i-1$. To complete the induction, we shall prove that the same holds for $B^{\prime} \in \mathfrak{B}(Q)$ with $\left|V\left(B^{\prime}\right)\right|=i$. Given such $B^{\prime}$, there exist $B \in \mathfrak{B}(Q)$ such that $B$ is obtained from $B^{\prime}$ by deleting all faces containing a free vertex of $B^{\prime}$. To be more precise, let $F V\left(B^{\prime}\right)=\left\{v_{i_{1}}, \ldots, v_{i_{r}}\right\}$ be the set of free vertices in $B^{\prime}$. Notice that regarding $B^{\prime}$ as a generic step of a retraction sequence in $\mathfrak{R}(Q)$, we can produce $r$ many different $B \in \mathfrak{B}(Q)$ with $|V(B)|=i-1$ from $B^{\prime}$. According to the induction hypothesis, we assume that for each $t=1, \ldots, r$, the group $H_{*}\left(\pi^{-1}\left(B\left(v_{i_{t}}\right)\right)\right)$ is concentrated in even degrees and torsion free, where $B\left(v_{i_{t}}\right) \in \mathfrak{B}(Q)$ is obtained from $B^{\prime}$ by deleting faces containing $v_{i_{t}}$. This assumption makes sense, because any retraction sequence reaches a union of edges.

For simplicity, we fix the following notation: For each free vertex $v_{i_{t}} \in F V\left(B^{\prime}\right)$,

- $X^{\prime}:=\pi^{-1}\left(B^{\prime}\right), \operatorname{dim} B^{\prime}=d^{\prime}=\frac{1}{2} \operatorname{dim}_{\mathbb{R}} X^{\prime}$.

- $X\left(v_{i_{t}}\right):=\pi^{-1}\left(B\left(v_{i_{t}}\right)\right), \operatorname{dim} B\left(v_{i_{t}}\right)=d=\frac{1}{2} \operatorname{dim}_{\mathbb{R}} X\left(v_{i_{t}}\right)$.

- $L\left(v_{i_{t}}\right):=L\left(\Delta_{E_{i_{t}}}\left(v_{i_{t}}\right), \xi_{E_{i_{t}}, v_{i_{t}}}\right)$, where $E_{i_{t}}$ denotes the maximal face of $B^{\prime}$ containing $v_{i_{t}}$.

Notice that $\operatorname{dim} L\left(v_{i_{t}}\right) \leq 2 d^{\prime}-1$ and $d \leq d^{\prime}$.

Now, we consider the following long exact sequence of the homology for the pair $\left(X^{\prime}, X\left(v_{i_{t}}\right)\right)=\left(\pi^{-1}\left(B^{\prime}\right), \pi^{-1}\left(B\left(v_{i_{t}}\right)\right)\right.$ :

$$
\begin{gathered}
\cdots \longrightarrow H_{j+1}\left(X^{\prime}\right) \longrightarrow H_{j+1}\left(X^{\prime}, X\left(v_{i_{t}}\right)\right) \longrightarrow \\
H_{j}\left(X\left(v_{i_{t}}\right)\right) \longrightarrow H_{j}\left(X^{\prime}\right) \longrightarrow H_{j}\left(X^{\prime}, X\left(v_{i_{t}}\right)\right) \longrightarrow
\end{gathered}
$$


Suppose that $j$ is odd. By the induction hypothesis and Lemma 4.2, the sequence (4.9) becomes

$$
0 \longrightarrow H_{j}\left(X^{\prime}\right) \longrightarrow \widetilde{H}_{j-1}\left(L\left(v_{i_{t}}\right)\right) \stackrel{0}{\longrightarrow} H_{j-1}\left(X\left(v_{i_{t}}\right)\right) .
$$

The map on the most right side is the zero map because the domain is a torsion group but the target space is free by assumption. Hence, $H_{j}\left(X^{\prime}\right)$ is isomorphic to $\widetilde{H}_{j-1}\left(L\left(v_{i_{t}}\right)\right)$, and the latter is zero if $j-1>\operatorname{dim} L\left(v_{i_{t}}\right)$ or a torsion group determined by the prime factors of $\left|G_{B^{\prime}}\left(v_{i_{t}}\right)\right|$ if $j-1 \leq \operatorname{dim} L\left(v_{i_{t}}\right)$ by Lemma 3.8. This argument holds for each free vertex $v_{i_{1}}, \ldots, v_{i_{r}}$. Hence we have $r$ many different exact sequences like (4.10). Now, the assumption of Theorem 1.1 tells us that

$$
\operatorname{gcd}\left\{\left|\widetilde{H}_{j-1}\left(L\left(v_{i_{1}}\right)\right)\right|, \ldots,\left|\widetilde{H}_{j-1}\left(L\left(v_{i_{r}}\right)\right)\right|\right\}=1,
$$

but $H_{j}\left(X^{\prime}\right)$ stays same. Hence, we conclude that $H_{j}\left(X^{\prime}\right)=0$ if $j$ is odd. Moreover, $\widetilde{H}_{j-1}\left(L\left(v_{i_{t}}\right)\right)=0$ for all $t=1, \ldots, r$ because of the exactness of (4.10).

Next, we assume that $j$ is even. Then, the exact sequence (4.9) gives us

$$
\widetilde{H}_{j}\left(L\left(v_{i_{t}}\right)\right) \stackrel{0}{\longrightarrow} H_{j}\left(X\left(v_{i_{t}}\right)\right) \longrightarrow H_{j}\left(X^{\prime}\right) \longrightarrow \widetilde{H}_{j-1}\left(L\left(v_{i_{t}}\right)\right) \longrightarrow 0 .
$$

Then, we have the following three cases:

$$
\begin{array}{ll}
\ldots \stackrel{0}{\longrightarrow} H_{j}\left(X\left(v_{i_{t}}\right)\right) \longrightarrow H_{j}\left(X^{\prime}\right) \longrightarrow 0, & \text { if } j-1>\operatorname{dim} L\left(v_{i_{t}}\right), \\
\cdots \stackrel{0}{\longrightarrow} H_{j}\left(X\left(v_{i_{t}}\right) \longrightarrow H_{j}\left(X^{\prime}\right) \longrightarrow \mathbb{Z} \longrightarrow 0,\right. & \text { if } j-1=\operatorname{dim} L\left(v_{i_{t}}\right), \\
\cdots \stackrel{0}{\longrightarrow} H_{j}\left(X\left(v_{i_{t}}\right)\right) \longrightarrow H_{j}\left(X^{\prime}\right) \longrightarrow G_{j-1} \longrightarrow 0, & \text { if } j-1<\operatorname{dim} L\left(v_{i_{t}}\right),
\end{array}
$$

where $G_{j-1}$ is defined in Lemma 3.8 and $H_{j}\left(X\left(v_{i_{t}}\right)\right)$ is free by the induction hypothesis. The free vertices $v_{i_{1}}, \ldots, v_{i_{r}}$ in $B^{\prime}$ gives us $r$ many exact sequences, and each of them is one of the above three cases. If one of the free vertices gives the first or the second type of exact sequence, then $H_{j}\left(X^{\prime}\right)$ cannot have a torsion subgroup because of the exactness. If all of the sequences are of the third type, then $H_{j}\left(X^{\prime}\right)$ has no torsion because of the assumption of the theorem and and arguments similar to those used in the case when $j$ is odd. This completes the induction.

Notice that Kawasaki in Kaw73] has shown that the cohomology ring of weighted projective space $\mathbb{C P}_{\chi}^{n}$ with weight $\chi=\left(\chi_{0}, \ldots, \chi_{n}\right)$ is concentrated in even degrees and torsion free, if $\operatorname{gcd}\left(\chi_{0}, \ldots, \chi_{n}\right)=1$. Theorem 1.1 extends Kawasaki's theorem to the category of toric orbifolds which contains the weighted projective spaces. The following Example 4.4 shows how we can apply this result to a polygon, and Example 4.5 is a practical computation on a higher dimensional weighed projective space.

Example 4.4. Consider the 4-dimensional toric orbifold $X$ over $Q$ whose $\mathcal{R}$ characteristic pair is described in Figure 4. Let $H(v)$ be an affine hyperplane defined in (3.9). Then $H(v) \cap Q$ is an 1-simplex. The induced $\mathcal{L}$-characteristic function

$$
\xi_{Q, v}:\left\{H(v) \cap F_{1}, H(v) \cap F_{m}\right\} \rightarrow \mathbb{Z}^{2}
$$

is defined by $\xi_{Q, v}\left(H(v) \cap F_{1}\right)=\lambda\left(F_{1}\right)=\left(a_{1}, b_{1}\right)$ and $\xi_{Q, v}\left(H(v) \cap F_{m}\right)=\lambda\left(F_{m}\right)=$ $\left(a_{m}, b_{m}\right)$. Therefore, the orbifold lens space $L\left(\Delta_{Q}(v), \xi_{Q, v}\right)$ is homeomorphic to $S^{3} / G_{Q}(v)$, where $G_{Q}(v)$ is a finite abelian group of order $\left|a_{1} b_{m}-b_{1} a_{m}\right|$, see Proposition 3.6. Moreover, the prime factors of the order of a torsion element 


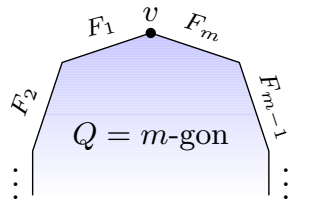

$$
\Lambda=\left[\begin{array}{cccc}
\lambda_{1} & \lambda_{2} & \cdots & \lambda_{4} \\
a_{1} & a_{2} & \cdots & a_{m} \\
b_{1} & b_{2} & \cdots & b_{m}
\end{array}\right]
$$

Figure 4. An $\mathcal{R}$-characteristic function on a polygon.

in $H_{*}\left(L\left(\Delta_{Q}(v), \xi_{Q, v}\right)\right)$ is a subset of the prime factors of $\left|a_{1} b_{m}-b_{1} a_{m}\right|$ by Lemma 3.8 .

Now, we consider a retraction sequence $\left\{B_{k}, E_{k}, b_{k}\right\}_{k=1}^{\ell}$ starting at $v$. The second space $B_{2}$ is the union $F_{2} \cup \cdots \cup F_{m-1}$ of edges whose preimage $\pi^{-1}\left(B_{2}\right)$ is homotopic to the wedge of $m-2$ copies of $\mathbb{C P}^{1}$. Hence, $H_{*}\left(\pi^{-1}\left(B_{2}\right)\right)$ is torsion free and $H_{\text {odd }}\left(\pi^{-1}\left(B_{2}\right)\right)$ vanishes. A cofibration

$$
L\left(\Delta_{Q}(v), \xi_{Q, v}\right) \longrightarrow \pi^{-1}\left(B_{2}\right) \longrightarrow X
$$

gives an isomorphism $H_{j}\left(X, \pi^{-1}\left(B_{2}\right)\right) \cong \widetilde{H}_{j-1}\left(L\left(\Delta_{Q}(v), \xi_{Q, v}\right)\right)$. Hence, the long exact sequence of pair $\left(X, \pi^{-1}\left(B_{2}\right)\right)$ yields

$\cdots \rightarrow \widetilde{H}_{j}\left(L\left(\Delta_{Q}(v), \xi_{Q, v}\right)\right) \rightarrow H_{j}\left(\pi^{-1}\left(B_{2}\right)\right) \rightarrow H_{j}(X) \rightarrow \widetilde{H}_{j-1}\left(L\left(\Delta_{Q}(v), \xi_{Q, v}\right)\right) \rightarrow \cdots$,

and this shows that, if $H_{j}(X)$ has a torsion part, then its prime factors must divide $\left|a_{1} b_{m}-b_{1} a_{m}\right|$. But, the same argument can be applied to all the other vertices in $Q$. Finally, we may conclude that $H_{*}(X)$ is torsion free and concentrated in even degrees, if

$$
\operatorname{gcd}\left\{\left|a_{1} b_{2}-b_{1} a_{2}\right|, \ldots,\left|a_{m-1} b_{m}-b_{m-1} a_{m}\right|,\left|a_{1} b_{m}-b_{1} a_{m}\right|\right\}=1,
$$

which is the assumption of Theorem 1.1 .

Example 4.5. We consider an $\mathcal{R}$-characteristic pair $\left(\Delta^{4}, \lambda\right)$, where $\lambda: \mathscr{F}\left(\Delta^{4}\right) \rightarrow$ $\mathbb{Z}^{4}$ is defined by

$$
\left.\begin{array}{ccccc}
\lambda_{1} & \lambda_{2} & \lambda_{3} & \lambda_{4} & \lambda_{5} \\
-1 & 1 & 0 & 0 & 0 \\
-2 & 0 & 1 & 0 & 0 \\
-2 & 0 & 0 & 1 & 0 \\
-2 & 0 & 0 & 0 & 1
\end{array}\right] .
$$

The column vectors satisfies the relation $\lambda_{1}+\lambda_{2}+2 \lambda_{3}+2 \lambda_{4}+2 \lambda_{5}=\mathbf{0}$. Then the resulting toric orbifold is a weighted projective space $\mathbb{C P}_{(1,1,2,2,2)}^{4}$. We refer to [Ful93, Section 2.2] or [CLS11, Example 3.1.17] for more details.

To check the assumption in Theorem 1.1. it suffices to consider all faces of $\Delta^{4}$, dimension greater than 1 , because the set $\mathfrak{B}\left(\Delta^{4}\right)$ coincides with the set of all faces of $\Delta^{4}$. First of all, for $\Delta^{4}$ itself, it is easy to see that

$$
\operatorname{gcd}\left\{\left|G_{\Delta^{4}}(v)\right| \mid v \in V\left(\Delta^{4}\right)\right\}=\operatorname{gcd}\{2,2,2,1,1\}=1
$$

Since the process is essentially same, we choose $E=F_{1} \cap F_{2}=\Delta^{2}$ as a sample. Observe that

$$
\begin{aligned}
\left(\left\langle\lambda_{1}, \lambda_{2}\right\rangle \otimes_{\mathbb{Z}} \mathbb{R}\right) \cap \mathbb{Z}^{4} & =\left(\left\langle-e_{1}-2 e_{2}-2 e_{3}-2 e_{4}, e_{1}\right\rangle \otimes_{\mathbb{Z}} \mathbb{R}\right) \cap \mathbb{Z}^{4} \\
& \cong\left\langle e_{2}+e_{3}+e_{4}, e_{1}\right\rangle .
\end{aligned}
$$




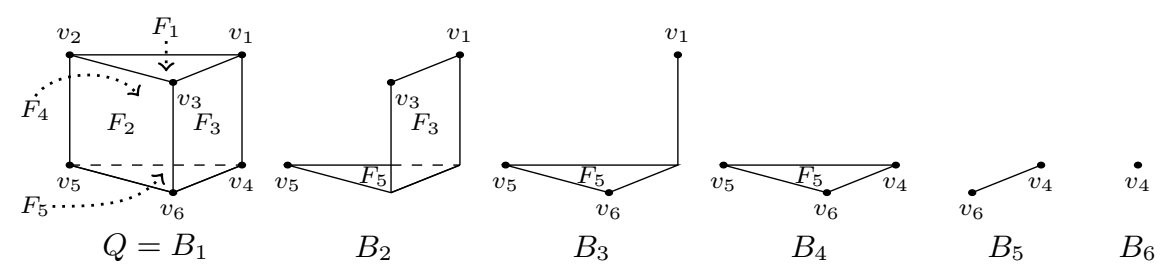

FiguRe 5. A retraction sequence of a prism

Hence, we may decompose the target space $\mathbb{Z}^{4} \cong\left\langle e_{2}+e_{3}+e_{4}\right\rangle \oplus\left\langle e_{1}\right\rangle \oplus\left\langle e_{3}\right\rangle \oplus\left\langle e_{4}\right\rangle$. This derives an $\mathcal{R}$-characteristic function

$$
\lambda_{E}:\left\{E \cap F_{3}, E \cap F_{4}, E \cap F_{5}\right\} \rightarrow \mathbb{Z}^{2} \cong\left\langle e_{3}\right\rangle \oplus\left\langle e_{4}\right\rangle,
$$

defined by $\lambda_{E}\left(E \cap F_{3}\right)=(-1,-1), \lambda_{E}\left(E \cap F_{4}\right)=(1,0)$ and $\lambda_{E}\left(E \cap F_{5}\right)=(0,1)$. Hence, $\pi^{-1}(E)=X\left(\Delta^{2}, \lambda_{E}\right) \cong \mathbb{C P}_{(1,1,1)}^{2}$. Hence, we have

$$
\operatorname{gcd}\left\{\left|G_{E}(v)\right| \mid v \in V(E)\right\}=\operatorname{gcd}\{1,1,1\}=1 .
$$

Sometimes, if the polytope has sufficiently many symmetries, we can analyze all possible retraction sequences efficiently. Proposition 4.3 can then be used to ensure the gcd assumption of Theorem 1.1 holds. The main features of the following example are that the polytope has at least 2 free vertices at each $B \in \mathfrak{B}(Q)$, and that the collection $\left\{\left|G_{Q}(v)\right| \mid v \in V(Q)\right\}$ consists of mutually different prime numbers, in particular, they are pairwise relatively prime.

Example 4.6. Let $Q$ be the 3 -dimensional cube whose facets and vertices are illustrated in Figure 5. We assign an $\mathcal{R}$-characteristic function $\lambda: \mathscr{F}(Q) \rightarrow \mathbb{Z}^{3}$ as follows;

$$
\lambda\left(F_{1}\right)=\left(p_{1}, p_{2}, p_{3}\right), \lambda\left(F_{5}\right)=\left(p_{4}, p_{5}, p_{6}\right), \lambda\left(F_{2}\right)=\mathbf{e}_{1}, \lambda\left(F_{3}\right)=\mathbf{e}_{2}, \lambda\left(F_{4}\right)=\mathbf{e}_{3},
$$

where $p_{i}$ 's are all prime numbers with $p_{i} \neq p_{j}$ whenever $i \neq j$, and $\mathbf{e}_{i}$ is the $i$-th standard unit vector in $\mathbb{Z}^{3}$. Then, it is easy to see that $\left|G_{Q}\left(v_{i}\right)\right|=p_{i}$, for $i=1, \ldots, 6$. Hence, we have

$$
\operatorname{gcd}\left\{\left|G_{Q}(v)\right| \mid v \in V(Q)\right\}=\operatorname{gcd}\left\{p_{1}, \ldots, p_{6}\right\}=1 .
$$

The same property holds for other polytopal complex $B \in \mathfrak{B}(Q)$ from Proposition 4.3. Indeed, for instance,

$$
\operatorname{gcd}\left\{\left|G_{B_{2}}(v)\right| \mid v \in F V\left(B_{2}\right)\right\}=\operatorname{gcd}\left\{\left|G_{B_{2}}\left(v_{1}\right)\right|,\left|G_{B_{2}}\left(v_{3}\right)\right|,\left|G_{B_{2}}\left(v_{5}\right)\right|\right\}=1
$$

because $\operatorname{gcd}\left\{p_{1}, p_{3}, p_{5}\right\}=1$.

\section{Cohomology Ring of TORIC ORBIFOldS}

The integral equivariant cohomology ring of certain projective toric varieties is given by a ring determined by the fan data. This ring is called the ring of piecewise polynomials which we denote by $\mathcal{P} \mathcal{P}[\Sigma]$. For a smooth fan, it uses the fan's combinatorial data only and coincides with the Stanley-Reisner ring $\mathcal{S R}[\Sigma]$ of the fan $\Sigma$. In general however, the ring of piecewise polynomials uses all the geometric data in a fan.

To be more precise, let $\Sigma$ be a fan in $\mathbb{R}^{n}$ and $\left\{\lambda_{1}, \ldots, \lambda_{m}\right\} \subset \mathbb{Z}^{n}$ the set of primitive vectors generating 1-dimensional rays in $\Sigma$. Then, the Stanley-Reisner 
ring $\mathcal{S R}[\Sigma]$ is defined by the quotient $\mathbb{Z}\left[x_{1}, \ldots, x_{m}\right] / \mathcal{I}$ of polynomial ring with $m$ variables by the following ideal generated by square free monomials:

$$
\left.\mathcal{I}=\left\langle x_{i_{1}} \cdots x_{i_{k}}\right| \text { cone }\left\{\lambda_{i_{1}}, \ldots, \lambda_{i_{k}}\right\} \notin \Sigma\right\rangle
$$

where cone $\left\{\lambda_{i_{1}}, \ldots, \lambda_{i_{k}}\right\}$ denotes the cone generated by $\left\{\lambda_{i_{1}}, \ldots, \lambda_{i_{k}}\right\}$. For the case of smooth toric varieties, their odd degree cohomology always vanishes, which leads us the following description of cohomology ring.

Theorem 5.1 (Dan78, Jur85). Let $X_{\Sigma}$ be a smooth toric variety. Then, there exists a ring isomorphism $H^{*}\left(X_{\Sigma}\right) \cong \mathcal{S} \mathcal{R}[\Sigma] / \mathcal{J}$, where $\mathcal{J}$ is the ideal generated by the linear relations

$$
\sum_{i=1}^{m}\left\langle\lambda_{i}, \mathbf{e}_{j}\right\rangle x_{i}=0, \quad j=1, \ldots, n
$$

where $\mathbf{e}_{j}$ denotes the $j$-th standard unit vector in $\mathbb{Z}^{n}$.

Notice that, for toric orbifolds, the theorem holds only for $\mathbb{Q}$-coefficients; see for instance, CLS11, Section 12.4]. In order to make the singular theory better resemble the smooth case, we introduce an intermediate ring, which models the Stanley-Reisner ring but is based on a fan $\widehat{\Sigma}$ in $\mathbb{R}^{m}$ defined from the combinatorial data of $\Sigma$, which has $m$ one-dimensional rays. The ring of piecewise polynomials on the original fan $\Sigma$ is recovered by imposing an integrality condition, which leads us the notion of the weighted Stanley-Reisner ring $w \mathcal{S R}[\Sigma]$ of $\Sigma$.

5.1. Weighted Stanley-Reisner ring. Let $\Sigma$ be a simplicial fan in $\mathbb{R}^{n}$, i.e., each top dimensional cone of $\Sigma$ is generated by $n$ linearly independent primitive vectors in the lattice $\mathbb{Z}^{n}$. In particular, a simplicial fan $\Sigma$ is called a polytopal fan if it is the normal fan of a simple lattice polytope in $\mathbb{R}^{n}$; see [CLS11, Chapter 2] or [Ful93, Section 1.5] for more details. Hence, the determinant of generators of each top dimensional cone is nonzero but not necessarily be \pm 1 , and so the corresponding fixed point might be singular. Let $\Sigma^{(j)}$ denotes the set of $j$-dimensional cones in $\Sigma$. To record the singularity of each fixed point in an efficient way, we assign a vector

$$
z^{\sigma}:=\left(z_{1}^{\sigma}, \ldots, z_{m}^{\sigma}\right) \in \bigoplus_{m} \mathbb{Q}\left[u_{1}, \ldots, u_{n}\right]
$$

to each top dimensional cone $\sigma=\operatorname{cone}\left\{\lambda_{i_{1}}, \ldots, \lambda_{i_{n}}\right\} \in \Sigma^{(n)}$ by the following rule:

(C1) $z_{j}^{\sigma}=0$ if $j \notin\left\{i_{1}, \ldots, i_{n}\right\}$,

$$
\left[\begin{array}{c}
z_{i_{1}}^{\sigma} \\
\vdots \\
z_{i_{n}}^{\sigma}
\end{array}\right]=\left[\begin{array}{l|l|l}
\lambda_{i_{1}} & \cdots & \lambda_{i_{n}}
\end{array}\right]^{-1} \cdot\left[\begin{array}{c}
u_{1} \\
\vdots \\
u_{n}
\end{array}\right]
$$

The inverse matrix in the condition (C2) may have rational entries. The following definition is motivated by this observation.

Definition 5.2. Given a fan $\Sigma$ in $\mathbb{R}^{n}$ with $m$ one-dimensional rays, we say a polynomial $h\left(x_{1}, \ldots, x_{m}\right) \in \mathbb{Z}\left[x_{1}, \ldots, x_{m}\right]$ satisfies the integrality condition with respect to $\Sigma$, if $h\left(z^{\sigma}\right) \in \mathbb{Z}\left[x_{1}, \ldots, x_{m}\right]$ for all $\sigma \in \Sigma^{(n)}$.

Notice that the collection of polynomials satisfying the integrality condition is closed under the addition and multiplication, which induces the natural ring structure on it inherited from that of $\mathbb{Z}\left[x_{1}, \ldots, x_{m}\right]$. Moreover, the polynomials in $\mathcal{I}$ 
defined in (5.1) satisfy the integrality condition because of the condition (C1). Finally, we define the weighted Stanley-Reisner ring $w \mathcal{S R}[\Sigma]$ as follows:

$$
w \mathcal{S R}[\Sigma]:=\left\{h \in \mathbb{Z}\left[x_{1}, \ldots, x_{m}\right] \mid h \text { satisfies the integrality condition }\right\} / \mathcal{I} \text {. }
$$

Remark 5.3. When the fan $\Sigma$ is smooth, $w \mathcal{S R}[\Sigma]=\mathcal{S R}[\Sigma]$. Indeed, the determinant of a smooth top dimensional cone is \pm 1 , which implies that its inverse has integer entries.

Now, we introduce the second main theorem of this paper. The proof will be given in the next subsection.

Theorem 5.4. Let $X_{\Sigma}$ be a projective toric orbifold over a polytopal fan $\Sigma$ with $H^{\text {odd }}(X)=0$. Then, there is a ring isomorphism

$$
H^{*}\left(X_{\Sigma}\right) \cong w \mathcal{S R}[\Sigma] / \mathcal{J},
$$

where $\mathcal{J}$ is the ideal generated by linear relations (5.2).

Consider a simple lattice polytope $Q$ in $\mathbb{R}^{n}$ whose normal fan is $\Sigma$. Then, the normal vectors of each facet define an $\mathcal{R}$-characteristic function $\lambda: \mathscr{F}(Q) \rightarrow \mathbb{Z}^{n}$. Now, we have a natural $\mathcal{R}$-characteristic pair $(Q, \lambda)$ from $\Sigma$, which allows us to apply the results of Section 2 and Section 4 . Hence, we have a concrete statement which is Theorem 1.2 with a sufficient condition for $H^{\text {odd }}\left(X_{\Sigma}\right)=0$.

We complete this subsection by applying Theorem 1.2 to a weighted projective space $\mathbb{C P}_{(1, a, b)}^{2}$. We shall recover the Kawasaki's result [Kaw73, Theorem 1].

Example 5.5. Let $\Sigma$ be a fan in $\mathbb{R}^{2}$ generated by

$$
\lambda_{1}=(a, b), \lambda_{2}=(-1,0), \lambda_{3}=(0,-1) \in \mathbb{Z}^{2}
$$

where $a$ and $b$ are relatively prime. The 2-dimensional cones are $\sigma_{12}, \sigma_{13}, \sigma_{23}$, where $\sigma_{i j}=$ cone $\left\{\lambda_{i}, \lambda_{j}\right\}$. Since $\left\{\lambda_{1}, \lambda_{2}, \lambda_{3}\right\}$ generates the lattice $\mathbb{Z}^{2}$ and satisfies $\lambda_{1}+a \lambda_{2}+b \lambda_{3}=(0,0)$, the toric variety $X_{\Sigma}$ is isomorphic to the weighed projective space $\mathbb{C P}_{(1, a, b)}^{2}$. We refer to [Ful93, Section 2.2] or [CLS11, Example 3.1.17] for the characterization of a fan corresponding to weighted projective spaces.

The direct computation of inverse matrices for $\left[\lambda_{i} \mid \lambda_{j}\right]$ gives us the following list of vectors:

$$
\begin{array}{rlr}
z^{\sigma_{12}}=\left(\begin{array}{rrr}
\frac{1}{b} u_{2}, & -u_{1}+\frac{a}{b} u_{2}, & 0
\end{array}\right), \\
z^{\sigma_{13}}=\left(\begin{array}{rrr}
\frac{1}{a} u_{1}, & 0, & \frac{b}{a} u_{1}-u_{2}
\end{array}\right), \\
z^{\sigma_{23}}=\left(\begin{array}{rrr}
0, & -u_{1}, & -u_{2}
\end{array}\right) .
\end{array}
$$

Hence, we have

$w \mathcal{S R}[\Sigma]=\left\{h\left(x_{1}, x_{2}, x_{3}\right) \in \mathbb{Z}\left[x_{1}, x_{2}, x_{3}\right] \mid h\left(z^{\sigma_{i j}}\right) \in \mathbb{Z}\left[u_{1}, u_{2}\right]\right.$, for $\left.1 \leq i<j \leq 3\right\} / \mathcal{I}$.

Finding elements at each degree is straightforward. For instance, a degree 2 polynomial $k_{1} x_{1}+k_{2} x_{2}+k_{3} x_{3} \in w \mathcal{S R}[\Sigma]$ if and only if the following three polynomials have integer coefficients:

(1) $-k_{2} u_{1}+\left(\frac{1}{b} k_{1}+\frac{a}{b} k_{2}\right) u_{2}$,

(2) $\left(\frac{1}{a} k_{1}+\frac{b}{a} k_{3}\right) u_{1}-k_{3} u_{2}$,

(3) $-k_{2} u_{1}-k_{2} u_{2}$, 
which is exactly the case when $k_{1}+a k_{2} \in b \mathbb{Z}$ and $k_{1}+b k_{3} \in a \mathbb{Z}$. Hence, one can show that the integers $\left(k_{1}, k_{2}, k_{3}\right)$ are

$$
(a,-1,0),(b, 0,-1),(a b, 0,0),(0, b, 0),(0,0, a),
$$

and $\mathbb{Z}$-linear combinations of them. They give us the following degree 2 elements in $w \mathcal{S R}[\Sigma]$,

$$
a x_{1}-x_{2}, b x_{1}-x_{3}, a b x_{1}, b x_{2}, a x_{3},
$$

and $\mathbb{Z}$-linear combinations of them. Similarly, we can find the degree 4 elements by

$$
a^{2} b^{2} x_{1}^{2}, b^{2} x_{2}^{2}, a^{2} x_{3}^{2}, a b x_{1} x_{2}, a^{2} x_{1} x_{3}, x_{2} x_{3},
$$

and $\mathbb{Z}$-linear combinations of them.

We continue to calculate the ring structure of $H^{*}\left(\mathbb{C P}_{(1, a, b)}^{2}\right)$ by Theorem 1.2 . Indeed, the $\mathcal{R}$-characteristic pair $\left(\Delta^{2}, \lambda\right)$ induced from $\Sigma$ satisfies the assumption of Theorem 1.1, see Example 4.4. Hence, we conclude that $\mathbb{C P}_{(1, a, b)}^{2}$ is even, which implies that the rank of integral cohomology group is 1 in each even degree and 0 otherwise.

Remark 5.6. In general, the integral betti numbers of a toric manifold or the rational betti numbers of a toric orbifold are given by the $h$-vector of underlying polytope, see [DJ91, Section 3] or [PS10, Section 4]. Hence, if a toric orbifold is even, then its integral betti numbers are obtained by the $h$-vector of the underlying polytope.

Now, the characteristic vectors (5.4) and the relation (5.2) determine the ideal $\mathcal{J}=\left\langle a x_{1}-x_{2}, b x_{1}-x_{3}\right\rangle$ whose generators are first two items in (5.6). Hence, the elements in (5.6) except first two are all eventually equal by $\mathcal{J}$ in $H^{*}\left(\mathbb{C P}_{(1, a, b)}^{2}\right)$. We put

$$
w_{1}:=a b x_{1}=b x_{2}=a x_{3} .
$$

Since $\operatorname{rank} H^{4}\left(\mathbb{C P}_{(1, a, b)}^{2}\right)=1$, we choose an element in (5.7) which has the minimal divisibility. In this case, we pick up

$$
w_{2}:=x_{2} x_{3} .
$$

Then, we have the multiplicative structure $w_{1}^{2}=a b w_{2}$. Finally, we have the following presentation

$$
H^{*}\left(\mathbb{C P}_{(1, a, b)}^{2}\right) \cong \mathbb{Z}\left[w_{1}, w_{2}\right] /\left\langle w_{1}^{2}-a b w_{2}, w_{1} w_{2}\right\rangle,
$$

where $\operatorname{deg} w_{1}=2, \operatorname{deg} w_{2}=4$. Notice that the monomial $w_{1} w_{2}$ comes from the Stanley-Reisner ideal $x_{1} x_{2} x_{3}$.

Remark 5.7. Even if we can find elements in $w \mathcal{S R}[\Sigma]$ by the direct computation of integrality condition, finding the minimal set of generators in $w \mathcal{S R}[\Sigma]$ for arbitrary simplicial fan is not obvious, in general. However, when $X_{\Sigma}$ is a weighted projective space, a result of [BFR09] allows us to find generators of the ring of piecewise polynomials $\mathcal{P} \mathcal{P}[\Sigma]$ and hence, generators in $w \mathcal{S} \mathcal{R}[\Sigma]$, by a method in the next subsection. Moreover, the identification result Corollary [5.11 tells us how to interprete those generators in terms of elements in $w \mathcal{S R}[\Sigma]$. 
5.2. Piecewise algebra and cohomology ring. We introduce now the ring of piecewise polynomials which is determined by a fan and describes the equivariant cohomology of a large class of toric orbifolds. As mentioned above, unlike the Stanley-Reisner ring, which encodes combinatorial data only, the ring of piecewise polynomials depends the full geometric information in a fan.

We begin by introducing piecewise polynomials. Let $\Sigma$ be a fan in $\mathbb{R}^{n}$. A function $f: \mathbb{Z}^{n} \rightarrow \mathbb{Z}$ is called a piecewise polynomial on $\Sigma$ if for each cone $\sigma \in \Sigma$ the restriction $\left.f\right|_{\sigma}$ is a polynomial function on $\sigma \cap \mathbb{Z}^{n}$. Such function can be interpreted as a collection $\left\{f_{\sigma}\right\}_{\sigma \in \Sigma^{(n)}}$, which we denote by $\left\{f_{\sigma}\right\}$ for simplicity, such that

$$
\left.f_{\sigma}\right|_{\sigma \cap \sigma^{\prime}}=\left.f_{\sigma^{\prime}}\right|_{\sigma \cap \sigma^{\prime}} .
$$

In other words, it is enough to consider the polynomials on each top dimensional cone. The polynomials on lower dimensional cones are determined by (5.8).

The set $\mathcal{P} \mathcal{P}[\Sigma]$ of piecewise polynomial functions on $\Sigma$ with integer coefficients on $\Sigma$ has a ring structure under the pointwise addition and multiplication. Moreover, the natural inclusion of global polynomials $\mathbb{Z}\left[u_{1}, \ldots, u_{n}\right]$ into $\mathcal{P} \mathcal{P}[\Sigma]$ induces a $\mathbb{Z}\left[u_{1}, \ldots, u_{n}\right]$-algebra structure on $\mathcal{P} \mathcal{P}[\Sigma]$. Furthermore, by considering $\mathbb{Q}^{n}$ instead of $\mathbb{Z}^{n}$, we can define piecewise polynomial functions with rational coefficients $f: \mathbb{Q}^{n} \rightarrow \mathbb{Q}$, and we denote the ring of piecewise polynomial functions with rational coefficients by $\mathcal{P} \mathcal{P}[\Sigma ; \mathbb{Q}]$.

It is well-known that the equivariant cohomology ring with rational coefficients of a toric variety over a simplicial fan is isomorphic to $\mathcal{P} \mathcal{P}[\Sigma ; \mathbb{Q}]$, see [CLS11. On the other hand, for the case of polytopal fans, Bahri, Franz and Ray [BFR09] proved the following proposition over $\mathbb{Z}$.

Proposition 5.8. BFR09, Proposition 2.2]. Let $\Sigma$ be a polytopal fan in $\mathbb{R}^{n}, X_{\Sigma}$ the associated compact projective toric variety with $H^{\text {odd }}\left(X_{\Sigma}\right)=0$, and $T=T^{n}$ the $n$-dimensional torus acting on $X_{\Sigma}$. Then, $H_{T}^{*}\left(X_{\Sigma}\right)$ is isomorphic to $\mathcal{P} \mathcal{P}[\Sigma]$ as an $H^{*}(B T)$-algebra .

Here, $H^{*}(B T)$-algebra structure on $\mathcal{P} \mathcal{P}[\Sigma]$ is obtained by identifying $H^{*}(B T)$ with the global polynomials $\mathbb{Z}\left[u_{1}, \ldots, u_{n}\right]$, where $u_{i}$ is the first Chern class of the canonical line bundle given by $i$-th projection $T \rightarrow S^{1}$.

On the other hand, the combinatorial structure of $\Sigma$ determines a canonical fan in a higher dimensional lattice as follows: Let $\Sigma^{(1)}=\left\{\lambda_{1}, \ldots, \lambda_{m}\right\}$ be the set of primitive vectors generating 1-dimensional rays in $\Sigma$. We define a linear map $\Lambda: \mathbb{Z}^{m} \rightarrow \mathbb{Z}^{n}$ by $\Lambda\left(\mathbf{e}_{i}\right)=\lambda_{i}$, where $\mathbf{e}_{1}, \ldots, \mathbf{e}_{m}$ denote the standard unit vectors in $\mathbb{Z}^{m}$. By the pull-back of $\Sigma$ through $\Lambda$, we can define a fan

$$
\widehat{\Sigma}=\left\{\hat{\sigma}:=\Lambda^{-1}(\sigma) \mid \sigma \in \Sigma\right\}
$$

in $\mathbb{R}^{m}$. To be more precise, if $\sigma$ is the cone generated by $\lambda_{i_{1}}, \ldots, \lambda_{i_{k}}$, then $\hat{\sigma}$ is the cone generated by $\mathbf{e}_{i_{1}}, \ldots, \mathbf{e}_{i_{k}}$. Moreover, for a commutative ring $\mathbf{k}$, a linear map $\Lambda$ induces a ring homomorphism

$$
\Lambda^{*}: \mathcal{P} \mathcal{P}[\Sigma ; \mathbf{k}] \rightarrow \mathcal{P} \mathcal{P}[\widehat{\Sigma} ; \mathbf{k}]
$$

of piecewise polynomial rings, where the map is defined by

$$
\Lambda^{*}\left(\left\{f_{\sigma}\right\}\right)=\left\{g_{\hat{\sigma}}\left(x_{i_{1}}, \ldots, x_{i_{n}}\right):=f_{\sigma}\left(\Lambda_{\sigma} \cdot\left[x_{i_{1}}, \ldots, x_{i_{n}}\right]^{T}\right)\right\}_{\hat{\sigma} \in \widehat{\Sigma}^{(n)}}
$$

where $\Lambda_{\sigma}=\left[\lambda_{i_{1}}|\ldots| \lambda_{i_{n}}\right]$ is a square matrix and

Indeed, the map $\Lambda^{*}$ is well-defined, since $\left.g_{\hat{\sigma}}\right|_{\hat{\sigma} \cap \hat{\sigma}^{\prime}}=\left.g_{\hat{\sigma}^{\prime}}\right|_{\hat{\sigma} \cap \hat{\sigma}^{\prime}}$. 
Lemma 5.9. Given a polytopal fan $\Sigma$, as $H^{*}(B T ; \mathbf{k})$-algebra:

(1) When $\mathbf{k}=\mathbb{Q}, \mathcal{P} \mathcal{P}[\Sigma ; \mathbb{Q}]$ is isomorphic to $\mathcal{P} \mathcal{P}[\widehat{\Sigma} ; \mathbb{Q}]$.

(2) When $\mathbf{k}=\mathbb{Z}$, there is a monomorphism from $\mathcal{P} \mathcal{P}[\Sigma]$ to $\mathcal{P} \mathcal{P}[\widehat{\Sigma}]$.

Proof. For each top dimensional cone $\sigma=\operatorname{cone}\left\{\lambda_{i_{1}}, \ldots, \lambda_{i_{n}}\right\} \in \Sigma^{(n)}$, we set the following notation:

- $f_{\sigma}\left(u_{1}, \ldots, u_{n}\right), g_{\hat{\sigma}}\left(x_{i_{1}}, \ldots, x_{i_{n}}\right)$ : polynomial functions defined on $\sigma \in \Sigma$ and $\hat{\sigma} \in \widehat{\Sigma}$, respectively.

- $\left\{f_{\sigma}\right\}:=\left\{f_{\sigma}\left(u_{1}, \ldots, u_{n}\right) \mid \sigma \in \Sigma^{(n)}\right\} \in \mathcal{P} \mathcal{P}[\Sigma]$.

- $\left\{g_{\hat{\sigma}}\right\}:=\left\{g_{\hat{\sigma}}\left(x_{i_{1}}, \ldots, x_{i_{n}}\right) \mid \hat{\sigma} \in \widehat{\Sigma}^{(n)}\right\} \in \mathcal{P} \mathcal{P}[\widehat{\Sigma}]$.

- $\Lambda_{\sigma}:=\left[\lambda_{i_{1}}|\cdots| \lambda_{i_{n}}\right]: n \times n$ matrix with column vectors $\lambda_{i_{1}}, \ldots, \lambda_{i_{n}}$.

Recall the ring homomorphism $\Lambda^{*}$ introduced in (5.9). If we restrict $\mathbf{k}$ to $\mathbb{Q}$, the map $\Lambda^{*}$ has the natural inverse

$$
\Theta: \mathcal{P P}[\widehat{\Sigma} ; \mathbf{k}] \rightarrow \mathcal{P} \mathcal{P}[\Sigma ; \mathbf{k}]
$$

defined by

$$
\Theta\left(\left\{g_{\hat{\sigma}}\right\}\right)=\left\{f_{\sigma}\left(u_{1}, \ldots, u_{n}\right):=g_{\hat{\sigma}}\left(\Lambda_{\sigma}^{-1} \cdot\left[u_{1}, \ldots, u_{n}\right]^{T} \mid \sigma \in \Sigma^{(n)}\right\},\right.
$$

where $\Lambda_{\sigma}^{-1}$ is regarded as a linear automorphism of $\mathbb{Q}^{n}$. Indeed,

$$
\begin{aligned}
\left(\Theta \circ \Lambda^{*}\right)\left(\left\{f_{\sigma}\right\}\right) & =\left\{f_{\sigma}\left(\Lambda_{\sigma} \cdot \Lambda_{\sigma}^{-1} \cdot\left[u_{1}, \ldots, u_{n}\right]^{T}\right) \mid \sigma \in \Sigma^{(n)}\right\} \\
& =\left\{f_{\sigma}\left(u_{1}, \ldots, u_{n}\right) \mid \sigma \in \Sigma^{(n)}\right\}=\left\{f_{\sigma}\right\} .
\end{aligned}
$$

In particular, $\Lambda^{*}$ is a monomorphism in $\mathbb{Z}$-coefficients. Finally, the $H^{*}(B T ; \mathbf{k})$ algebra structure on $\mathcal{P} \mathcal{P}[\widehat{\Sigma} ; \mathbf{k}]$ is naturally inherited from that of $\mathcal{P} \mathcal{P}[\Sigma ; \mathbf{k}]$ via the $\operatorname{map} \Lambda^{*}$.

Recall that the Stanley-Reisner ring $\mathcal{S} \mathcal{R}[\Sigma ; \mathbf{k}]$ has combinatorial data only, while $\mathcal{P} \mathcal{P}[\Sigma ; \mathbf{k}]$ contains both combinatorial and geometric data. However, $\mathcal{P} \mathcal{P}[\widehat{\Sigma} ; \mathbf{k}]$ has only combinatorics, but looks like $\mathcal{P} \mathcal{P}[\Sigma ; \mathbf{k}]$. In this point of view, $\mathcal{P} \mathcal{P}[\widehat{\Sigma} ; \mathbf{k}]$ is an intermediate object between $\mathcal{S R}[\Sigma ; \mathbf{k}]$ and $\mathcal{P} \mathcal{P}[\Sigma ; \mathbf{k}]$. The following lemma together with Lemma 5.9 concludes the relations among those three objects.

Lemma 5.10. As $H^{*}(B T ; \mathbf{k})$-algebra, $\mathcal{P} \mathcal{P}[\widehat{\Sigma} ; \mathbf{k}]$ is isomorphic to $\mathcal{S R}[\Sigma ; \mathbf{k}]$ for $\mathbf{k}=$ $\mathbb{Z}$ or $\mathbb{Q}$.

Proof. We construct an isomorphism between $\mathcal{P} \mathcal{P}[\widehat{\Sigma} ; \mathbf{k}]$ and $\mathcal{S} \mathcal{R}[\Sigma ; \mathbf{k}]$, where $\mathbf{k}=$ $\mathbb{Z}$ or $\mathbb{Q}$. Assume that $\left|\Sigma^{(1)}\right|=m$. Define a map

$$
\phi: \mathbf{k}\left[x_{1}, \ldots, x_{m}\right] \rightarrow \mathcal{P} \mathcal{P}[\widehat{\Sigma} ; \mathbf{k}]
$$

by restriction to each cone of $\widehat{\Sigma}$. Then, this map $\phi$ is surjective ring homomorphism. Indeed, given $\left\{g_{\hat{\sigma}}\right\} \in \mathcal{P} \mathcal{P}[\widehat{\Sigma} ; \mathbf{k}]$, we can apply the inclusion-exclusion principle to obtain

$$
h\left(x_{1}, \ldots, x_{m}\right)=\sum_{j=0}^{n-1}\left((-1)^{j} \sum_{\substack{\hat{\tau} \in \widehat{\Sigma} \\ \operatorname{dim} \hat{\tau}=n-j}} g_{\hat{\tau}}\left(x_{i_{1}}, \ldots, x_{i_{n-j}}\right)\right)
$$

which is the desired global function $h$ satisfying $\phi(h)=\left\{g_{\hat{\sigma}}\right\}$, where $\hat{\sigma} \in \widehat{\Sigma}^{(n)}$. 
Moreover, since the zero element in $\mathcal{P P}[\widehat{\Sigma} ; \mathbf{k}]$ is $\left\{g_{\hat{\sigma}}=0 \mid \hat{\sigma} \in \widehat{\Sigma}^{(n)}\right\}$, the kernel is

$$
\operatorname{ker} \phi=\operatorname{span}\left\{\prod_{j=1}^{k} x_{i_{j}} \mid \operatorname{cone}\left\{\mathbf{e}_{i_{1}}, \ldots, \mathbf{e}_{i_{k}}\right\} \notin \widehat{\Sigma}\right\},
$$

which is exactly the Stanley-Reisner ideal $\mathcal{I}$ of $\Sigma$. Hence, the result follows.

Corollary 5.11. There exists an isomorphism $\mathcal{P} \mathcal{P}[\Sigma] \cong w \mathcal{S R}[\Sigma]$ (see (5.31) as $H^{*}(B T)$-algebra.

Proof. Cosider the composition of ring homomorphisms

$$
\mathcal{P} \mathcal{P}[\Sigma] \stackrel{\Lambda^{*}}{\longrightarrow} \mathcal{P} \mathcal{P}[\widehat{\Sigma}] \stackrel{\Phi^{-1}}{\longrightarrow} \mathcal{S R}[\Sigma]
$$

where $\Phi: \mathcal{S R}[\Sigma] \rightarrow \mathcal{P} \mathcal{P}[\widehat{\Sigma}]$ is the isomorphism induced by $\phi$. With $\mathbb{Z}$-coefficients, the map $\Lambda^{*}$ is injective by Lemma 5.9. Hence, $\mathcal{P} \mathcal{P}[\Sigma]$ is isomorphic to its image in $\mathcal{S R}[\Sigma]$ via the composition $\Phi^{-1} \circ \Lambda^{*}$.

Recall that the composition $\Phi^{-1} \circ \Lambda^{*}$ is isomorphism over $\mathbb{Q}$, whose inverse $\Theta \circ \Phi^{-1}$ maps an element $[h] \in \mathcal{S R}[\Sigma ; \mathbb{Q}]$ to $\left\{h\left(z^{\sigma}\right)\right\}_{\sigma \in \Sigma^{(n)}} \in \mathcal{P} \mathcal{P}[\Sigma ; \mathbb{Q}]$. Therefore, over integer coefficients, $[h] \in \operatorname{im}\left(\Phi^{-1} \circ \Lambda^{*}\right)$ if and only if the polynomial $h$ satisfies the integrality condition. Hence, the result follows.

Finally, we conclude this subsection with a proof of Theorem 5.4

Proof of theorem 5.4. Since $H^{*}\left(X_{\Sigma} ; \mathbb{Z}\right)$ concentrated in even degrees, the Serre spectral sequence for the fibration

$$
X_{\Sigma} \longrightarrow E T \times_{T} X_{\Sigma} \stackrel{\pi}{\longrightarrow} B T
$$

degenerates at $E_{2}$ level. By the result from Franz and Puppe ([FP07, Theorem 1.1 ), we get the following isomorphisms of $H^{*}(B T)$-algebras,

$$
\begin{aligned}
H^{*}\left(X_{\Sigma}\right) & \cong H_{T}^{*}\left(X_{\Sigma}\right) \otimes_{H^{*}(B T)} \mathbb{Z} \\
& \cong H_{T}^{*}\left(X_{\Sigma}\right) / \operatorname{Im}\left(\pi^{*}: H^{*}(B T) \rightarrow H_{T}^{*}\left(X_{\Sigma}\right)\right) .
\end{aligned}
$$

By Proposition 5.8 and Corollary 5.11, we have $H_{T}^{*}\left(X_{\Sigma}\right) \cong w \mathcal{S R}[\Sigma]$. Moreover, for each $u_{j} \in \mathbb{Z}\left[u_{1}, \ldots, u_{n}\right] \cong H^{*}(B T)$,

$$
\left(\Phi \circ \Lambda^{*}\right)\left(u_{j}\right)=\sum_{i=1}^{m}\left\langle\lambda_{i}, e_{j}\right\rangle x_{i} .
$$

Hence, we conclude that $\operatorname{im}\left(\pi^{*}: H^{*}(B T) \rightarrow H_{T}^{*}\left(X_{\Sigma}\right)\right)=\mathcal{J}$.

\section{Example: Orbifold Hirzebruch varieties}

We finish this paper by illustrating the results of the previous sections by a concrete example which is not a weighted projective space. Consider a primitive vector $(a, b) \in \mathbb{Z}^{2}$ with $a>0$. Together with $(-1,0),(0,1),(0,-1)$, we can make a complete fan $\Sigma$ in $\mathbb{R}^{2}$ which gives us a compact toric variety with two singular points. We denote this toric variety by $\mathscr{H}_{(a, b)}$. See Figure 6 for the fan and corresponding $\mathcal{R}$-characteristic pair $(Q, \lambda)$. When $a=1$, the toric variety is known as a Hirzebruch surface, say $\mathscr{H}_{b}$. In this point of view, let us call $\mathscr{H}_{(a, b)}$ an orbifold Hirzebruch variety. 
Since the collection in (1.1) becomes $\left\{\left|G_{Q}(v)\right| \mid v \in V(Q)\right\}=\{1,1, a, a\}$ when $B_{1}=Q$, its gcd is 1 . Moreover, in any retraction sequence, $B_{2}$ is given by a union of edges, which guarantees that $(Q, \lambda)$ satisfies the assumption of Theorem [1.1 see Example 4.4. Moreover, since the underlying polytope is a square, the integral betti numbers are given by $\beta^{0}=\beta^{4}=1$ and $\beta^{2}=2$ by Remark 5.6 .

Remark 6.1. We may compute the (co)homology groups of low dimensional toric orbifolds by the spectral sequence whose $E_{1}$ page is described by the fan data; see Jor98 and Fis92. More generally, the low dimensional calculations of Kuwata, Masuda and Zeng [KMZ15] apply to the category of torus orbifolds.

Let $\sigma_{i j}=\operatorname{cone}\left\{\lambda_{i}, \lambda_{j}\right\}$, where $\lambda_{1}, \ldots, \lambda_{4}$ are described in the right hand side of Figure 6. Then, the integrality condition of Definition 5.2 is given by the following vectors:

$$
\begin{aligned}
& z^{\sigma_{12}}=\left(\begin{array}{llll}
\frac{1}{a} u_{1}, & -\frac{b}{a} u_{1}+u_{2}, & 0, & 0
\end{array}\right)
\end{aligned}
$$

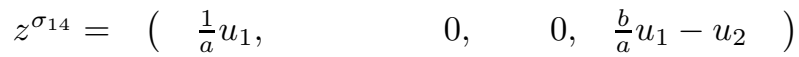

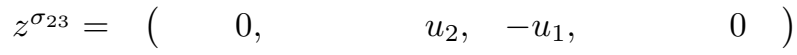

$$
\begin{aligned}
& z^{\sigma_{34}}=\left(\begin{array}{cccc}
0, & 0, & -u_{1}, & -u_{2}
\end{array}\right) .
\end{aligned}
$$

Notice that the last two vectors $z^{\sigma_{23}}$ and $z^{\sigma_{34}}$ don't contribute the integrality condition, because their entries have integral coefficients.

A similar computation to Example [5.5] shows that the following polynomials are elements of degree 2 in $w \mathcal{S R}[\Sigma]$ :

$$
a x_{1}-x_{3}, b x_{1}+x_{2}-x_{4}, a x_{1}, a x_{2}, x_{3}, a x_{4}
$$

as are $\mathbb{Z}$-linear combinations of them. The first two elements are actually the linear relations in $\mathcal{J}$, which means that they come from the global polynomials in $\mathcal{P} \mathcal{P}[\Sigma]$. Since $\operatorname{rank} H^{2}\left(\mathscr{H}_{(a, b)}\right)=2$, we choose two linearly independent elements as follows,

$$
w_{1}:=a x_{1} \text { and } w_{2}:=a x_{4} .
$$

Next, degree 4 elements in $w \mathcal{S R}[\Sigma]$ are

$$
a^{2} x_{1}^{2}, a^{2} x_{2}^{2}, x_{3}^{2}, a^{2} x_{4}^{2}, a^{2} x_{1} x_{2}, a^{2} x_{1} x_{4}, x_{2} x_{3} \text { and } x_{3} x_{4} \text {, }
$$

and their $\mathbb{Z}$-linear combination. First four of (6.2) are just the square of degree 2 elements. The remaining four monomials are:

- $a^{2} x_{1} x_{2}=a x_{1} a x_{2}=a x_{1} a\left(-b x_{1}+x_{4}\right)=w_{1}\left(-b w_{1}+w_{2}\right)$,

- $a^{2} x_{1} x_{4}=a x_{1} a x_{4}=w_{1}^{2}$,

Notice that the final two monomials $x_{2} x_{3}, x_{3} x_{4}$ cannot come from degree 2 elements. Hence, we put

$$
w_{3}:=x_{3} x_{4}
$$

Then,

$$
x_{2} x_{3}=\left(-b x_{1}+x_{4}\right) x_{3}=x_{3} x_{4}=a x_{1} x_{4}=a w_{3} .
$$



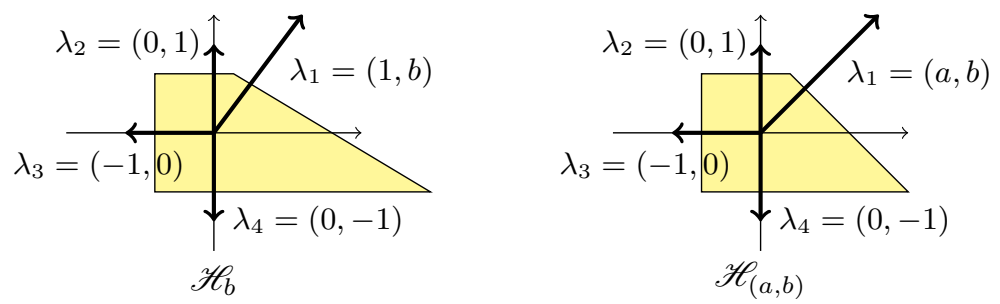

FiguRE 6. A Hirzebruch surface and an orbifold Hirzebruch variety.

The second equality holds because of the Stanley-Reisner ideal $\mathcal{I}=\left\langle x_{1} x_{3}, x_{2} x_{4}\right\rangle$. Finally, the ideal $\mathcal{I}$ and $\mathcal{J}$ determine the multiplicative structures as follows:

$$
\begin{aligned}
w_{1}^{2} & =\left(a x_{1}\right)^{2}=\left(a x_{1}\right)\left(x_{3}\right)=0, \\
w_{1} w_{2} & =\left(a x_{1}\right)\left(a x_{4}\right)=x_{3}\left(a x_{4}\right)=a w_{3}, \\
w_{2}^{2} & =\left(a x_{4}\right)\left(a x_{4}\right)=a\left(b x_{1}+x_{2}\right)\left(a x_{4}\right)=a b x_{3} x_{4}=a b w_{3}, \\
w_{1} w_{3} & =\left(a x_{1}\right)\left(x_{3} x_{4}\right)=0, \\
w_{2} w_{3} & =\left(a x_{4}\right)\left(x_{3} x_{4}\right)=a x_{4} x_{3}\left(b x_{1}+x_{2}\right)=0, \\
w_{3}^{2} & =\left(x_{3} x_{4}\right)^{2}=x_{3}^{2} x_{4}^{2}=\left(a x_{1} x_{3}\right)\left(x_{4}^{2}\right)=0 .
\end{aligned}
$$

Therefore, for the cohomology of the Hirzebruch variety, we we get the following presentation.

$$
H^{*}\left(\mathscr{H}_{(a, b)}\right) \cong \mathbb{Z}\left[w_{1}, w_{2}, w_{3}\right] /\left\langle w_{1}^{2}, w_{1} w_{2}-a w_{3}, w_{2}^{2}-a b w_{3}, w_{1} w_{3}, w_{2} w_{3}, w_{3}^{2}\right\rangle,
$$

where $\operatorname{deg} w_{1}=\operatorname{deg} w_{2}=2$ and $\operatorname{deg} w_{3}=4$.

Remark 6.2. The cohomology ring of Hirzebruch surface, by way of comparison, can be computed from the result of [Dan78, Jur85] or DJ91. Indeed it has the following presentation

$$
H^{*}\left(\mathscr{H}_{b}\right) \cong \mathbb{Z}\left[w_{1}, w_{2}\right] /\left(w_{1}^{2}, w^{2}-b w_{1} w_{2}\right),
$$

where $\operatorname{deg} w_{1}=\operatorname{deg} w_{2}=2$, which means that it is generated by degree 2 elements. However, $H^{*}\left(\mathscr{H}_{(a, b)}\right)$ has the degree 4 generator $w_{3}$ which cannot be generated by degree 2 elements, i.e., $w_{1} w_{2}=a w_{3}$. Notice that we can recover the presentation of $H^{*}\left(\mathscr{H}_{b}\right)$ by replacing $a$ into 1 in (6.3).

\section{REFERENCES}

[BFR09] Anthony Bahri, Matthias Franz, and Nigel Ray, The equivariant cohomology ring of weighted projective space, Math. Proc. Cambridge Philos. Soc. 146 (2009), no. 2, 395-405.

[Bor60] Armand Borel, Seminar on transformation groups, With contributions by G. Bredon, E. E. Floyd, D. Montgomery, R. Palais. Annals of Mathematics Studies, No. 46, Princeton University Press, Princeton, N.J., 1960.

[BP02] Victor M. Buchstaber and Taras E. Panov, Torus actions and their applications in topology and combinatorics, University Lecture Series, vol. 24, American Mathematical Society, Providence, RI, 2002.

[BSS] Anthony Bahri, Soumen Sarkar, and Jongbaek Song, Shellability and admissible retraction, In preparation (2016).

[Buc08] Weronika Buczynska, Fake weighted projective spaces, arXiv:0805.1211 v1 [math.AG] (2008). 
[CLS11] David A. Cox, John B. Little, and Henry K. Schenck, Toric varieties, Graduate Studies in Mathematics, vol. 124, American Mathematical Society, Providence, RI, 2011.

[Dan78] V. I. Danilov, The geometry of toric varieties, Uspekhi Mat. Nauk 33 (1978), no. 2(200), 85-134, 247.

[DJ91] Michael W. Davis and Tadeusz Januszkiewicz, Convex polytopes, Coxeter orbifolds and torus actions, Duke Math. J. 62 (1991), no. 2, 417-451.

[DKS] Alastair Darby, Shintaro Kuroki, and Jongbaek Song, The equivariant cohomology of torus orbifolds, In preparation.

[Fis92] Stephan Fischli, On toric varieties, Ph.D. thesis, Universität Bern (1992).

[FP07] Matthias Franz and Volker Puppe, Exact cohomology sequences with integral coeffcients for torus actions, Transform. Groups 12 (2007), no. 1, 65-76.

[Ful93] William Fulton, Introduction to toric varieties, Annals of Mathematics Studies, vol. 131, Princeton University Press, Princeton, NJ, 1993, The William H. Roever Lectures in Geometry.

[HHH05] Megumi Harada, André Henriques, and Tara S. Holm, Computation of generalized equivariant cohomologies of Kac-Moody flag varieties, Adv. Math. 197 (2005), no. 1, 198-221.

[HRHW15] Megumi Harada, Nigel Ray, Tara S. Holm, and Gareth Williams, The equivariant $k$-theory and cobordism rings of divisive spaces, To appear, Tohoku Math. J. (2015), no. arXiv:1306.1641 v3 [math.AT] (2013).

[Jor98] Arno Jordan, Homology and cohomology of toric varieties, Ph.D. thesis, University of Konstanz (1998).

[Jur85] Jerzy Jurkiewicz, Torus embeddings, polyhedra, $k^{*}$-actions and homology, Dissertationes Math. (Rozprawy Mat.) 236 (1985), 64.

[Kas09] Alexander M. Kasprzyk, Bounds on fake weighted projective space, Kodai Math. J. 32 (2009), no. 2, 197-208.

[Kaw73] T. Kawasaki, Cohomology of twisted projective spaces and lens complexes, Math. Ann. 206 (1973), 243-248.

[KMZ15] Hideya Kuwata, Mikiya Masuda, and Haozhi Zeng, Torsions in the cohomology of torus orbifolds, arXiv:1604.03138 (2015).

[PS10] Mainak Poddar and Soumen Sarkar, On quasitoric orbifolds, Osaka J. Math. 47 (2010), no. 4, 1055-1076.

[SS13] Soumen Sarkar and Dong Youp Suh, A new construction of lens spaces, arXiv:1304.4836 [math.AT] (2013).

[SU] Soumen Sarkar and Vikraman Uma, Equivariant k-theory and cobordism rings of toric orbifolds, In preparation.

[Zie95] Günter M. Ziegler, Lectures on polytopes, Graduate Texts in Mathematics, vol. 152, Springer-Verlag, New York, 1995.

Department of Mathematics, Rider University

E-mail address: bahri@rider.edu

Department of Mathematics, Indian Institute of Technology Madras

E-mail address: soumensarkar20@gmail.com

Department of Mathematical Sciences, Kaist

E-mail address: jongbaek.song@gmail.com 